\title{
GASDYNAMICS IN NGC 5248: FUELING A CIRCUMNUCLEAR STARBURST RING OF SUPER-STAR CLUSTERS
}

\author{
Shardha JoGeE \\ Division of Physics, Mathematics, and Astronomy, MS 105-24, California Institute of Technology, Pasadena, CA 91125; sj@pa.uky.edu \\ IsAAC SHLOSMAN ${ }^{1,2}$ \\ JILA, University of Colorado, Campus Box 440, Boulder, CO 80309-0440; shlosman@pa.uky.edu
}

SePpo Laine

Space Telescope Science Institute, 3700 San Martin Drive, Baltimore, MD 21218; laine@stsci.edu

Peter Englmaier

Max-Planck-Institut für extraterrestrische Physik, Postfach 1312,85741 Garching, Germany; ppe@mpe.mpg.de

JOHAN H. KNAPEN

Isaac Newton Group of Telescopes, Apartado 321, Santa Cruz de La Palma, Canary Islands E-38700, Spain; and Department of Physical Sciences, University of Hertfordshire, Hatfield, Herts AL10 9AB, UK; knapen@ing.iac.es

Nick SCOVILLE

Division of Physics, Mathematics, and Astronomy, MS 105-24, California Institute of Technology, Pasadena, CA 91125; nzs@astro.caltech.edu

AND

Christine D. WiLson

Department of Physics and Astronomy, McMaster University, Hamilton, ON L8S 4M1, Canada; wilson@physics.mcmaster.ca Received 2002 February 13; accepted 2002 April 12

\begin{abstract}
Through observations and modeling, we demonstrate how the recently discovered large-scale bar in NGC 5248 generates spiral structure that extends from $10 \mathrm{kpc}$ down to $100 \mathrm{pc}$, fuels star formation on progressively smaller scales, and drives disk evolution. Deep inside the bar, two massive molecular spirals cover nearly $180^{\circ}$ in azimuth, show streaming motions of $20-40 \mathrm{~km} \mathrm{~s}^{-1}$, and feed a starburst ring of super-star clusters at $375 \mathrm{pc}$. They also connect to two narrow $K$-band spirals that delineate the UV bright star clusters in the ring. The data suggest that the $K$-band spirals are young, and the starburst has been triggered by a bar-driven spiral density wave (SDW). The latter may even have propagated closer to the center where a second $\mathrm{H} \alpha$ ring and a dust spiral are found. The molecular and Hubble Space Telescope data support a scenario where stellar winds and supernovae efficiently clear out gas from dense star-forming regions on timescales less than a few Myr. We have investigated the properties of massive CO spirals within the framework of bar-driven SDWs, incorporating the effect of gas self-gravity. We find good agreement between the model predictions and the observed morphology, kinematics, and pitch angle of the spirals. This combination of observations and modeling provides the best evidence to date for a strong dynamical coupling between the nuclear region and the surrounding disk. It also confirms that a low central mass concentration, which may be common in late-type galaxies, is particularly favorable to the propagation of a bar-driven gaseous SDW deep into the central region of the galaxy, whereas a large central mass concentration favors other processes, such as the formation and decoupling of nuclear bars.
\end{abstract}

Subject headings: galaxies: evolution — galaxies: individual (NGC 5248) — galaxies: ISM — galaxies: kinematics and dynamics — galaxies: starburst — galaxies: structure

On-line material: color figures

\section{INTRODUCTION}

The level of activity and the rate of evolution in the circumnuclear region of galaxies depend to a large degree on dynamical processes that transport molecular gas. Stellar bars and other nonaxisymmetries are destined to drive the gas toward the central kiloparsec (Simkin, Su, \& Schwarz 1980; Shlosman, Frank, \& Begelman 1989; Athanassoula 1992). In some cases, stellar bars can be induced by tidal interactions (e.g., Noguchi 1988). The secular evolution of disk galaxies, therefore, proceeds in the direction of increas-

\footnotetext{
${ }^{1}$ JILA Visiting Fellow.

2 Permanent address: Department of Physics and Astronomy, University of Kentucky, Lexington, KY 40506-0055.
}

ing central mass concentration in the baryonic material (e.g., Pfenniger 1996).

The dynamical and secular evolution in the central kiloparsec, including stellar and nonstellar activities, depends on the degree of nonaxisymmetry in this region. Strongly nonaxisymmetric structures will increase the rate of evolution by facilitating the transfer of angular momentum and mass (e.g., Shlosman 2001). Observations of the central regions in barred galaxies provide clear evidence for nonaxisymmetries such as nuclear spiral structure, triaxial bulges, and nuclear (secondary) bars (e.g., Laine et al. 1999b; Jogee, Kenney, \& Smith 1999; Regan \& Mulchaey 1999; Martini \& Pogge 1999; Bureau 2002; Laine et al. 2002). Numerical simulations do reproduce much of this morphology, but fundamental issues remain, such as the state of the molecular gas 
in the central kiloparsec, the formation and dynamical decoupling of nested bars, the stellar dynamics and gasdynamics in decoupled systems, and the interactions of bars with dark matter halos (e.g., Friedli \& Martinet 1993; Jogee et al. 1999; Maciejewski \& Sparke 2000; Hüttemeister \& Aalto 2001; Shlosman \& Heller 2002; Athanassoula 2002; Shlosman 2002).

Both theory and observations reveal the importance of inner Lindblad resonances (ILRs) in slowing down the radial gas inflow within the inner kiloparsec of barred galaxies (e.g., Schwarz 1984; Combes \& Gerin 1985; Shlosman et al. 1989; Athanassoula 1992; Sofue 1991; Kenney et al. 1992; Knapen et al. 1995a, 1995b; Jogee 2001; Jogee et al. 2001). The resulting concentration of molecular gas leads to the formation of nuclear rings on scales of a few hundred parsecs (e.g., review by Buta \& Combes 1996). These rings exhibit elevated rates of star formation (SF) as a result of the gas becoming gravitationally unstable (e.g., Elmegreen 1994; Knapen et al. 1995a; Jogee 1999; Jogee et al. 2001) and are dynamically important (Heller \& Shlosman 1996).
The nearby $(D=15 \mathrm{Mpc})$ grand-design spiral galaxy NGC 5248 is a great laboratory for investigating gasdynamics, barred and spiral morphologies, and their impact on circumnuclear SF. It shows spectacular grand-design spiral structure that extends over a large dynamic range in radius, from $\sim 10 \mathrm{kpc}$ to $100 \mathrm{pc}$, and winds through a large angle $\left(>360^{\circ}\right)$ in azimuth. NGC 5248, with a revised Hubble type SAB(rs)bc (de Vaucouleurs et al. 1991), was until recently considered to host a short bar with a semimajor axis of $22^{\prime \prime}$ (1.6 kpc; Martin 1995) or even treated as unbarred (e.g., Patsis, Grosbol, \& Hiotelis 1997). However, Jogee et al. (2002, hereafter Paper I) have shown from a deep $R$-band image (Fig. 1), as well as from dynamical and photometric analyses, that the spiral structure is being driven by an extended, moderately strong stellar bar that has a deprojected ellipticity of 0.44 and a semimajor axis $a_{\mathrm{bar}}$ of $\sim 95^{\prime \prime}$ $(7.1 \mathrm{kpc})$. The bar is embedded within a fainter outer disk that is visible out to a radius of $230^{\prime \prime}(17.2 \mathrm{kpc})$. Previous optical and near-infrared (NIR) images did not have the sensitivity and/or field of view to clearly detect the outer

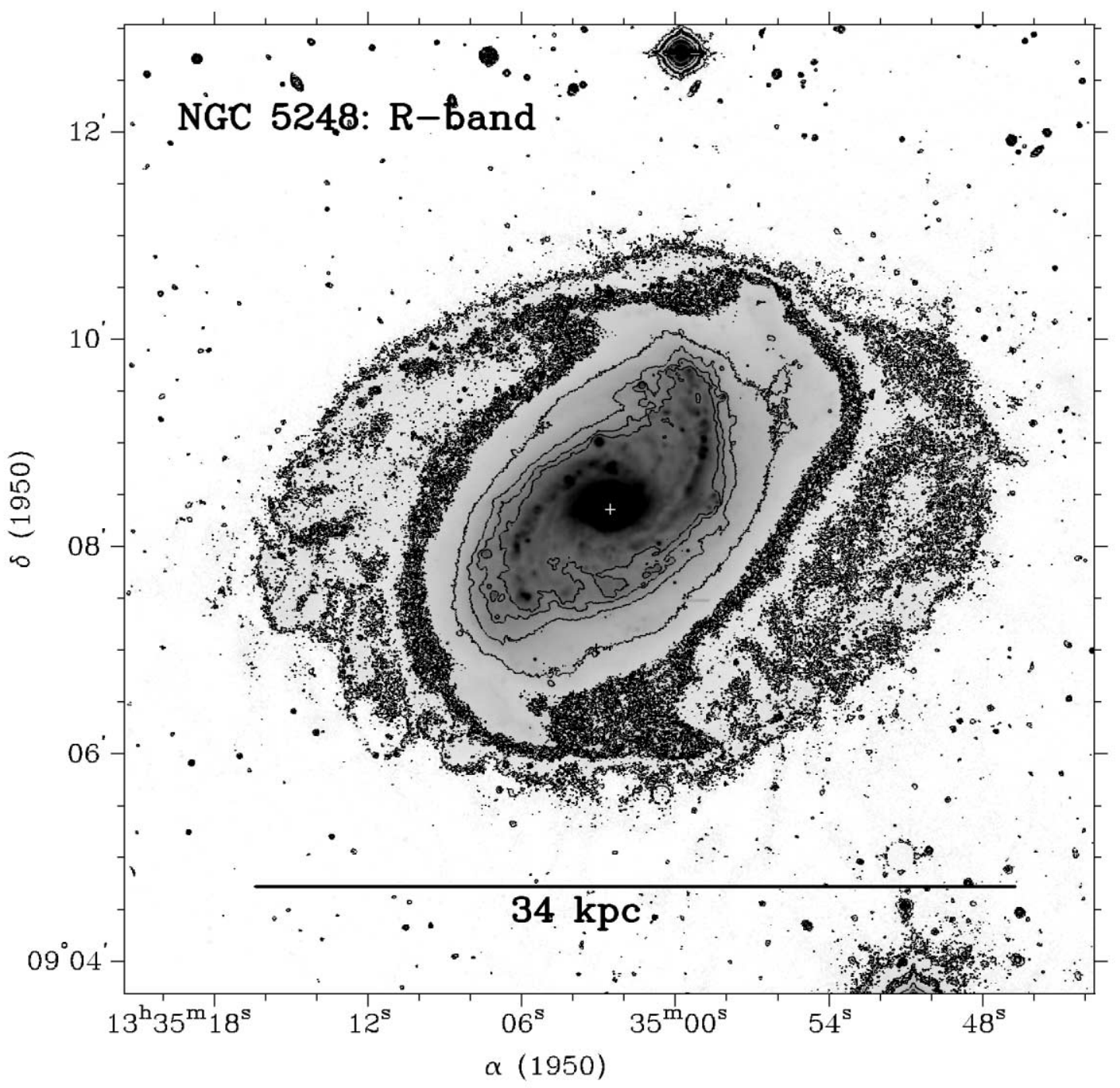

FIG. 1.-Large-scale stellar bar. The deep wide-field $R$-band image from Jogee et al. (2002) reveals a hitherto unknown prominent stellar bar with a semimajor axis of $\sim 95^{\prime \prime}(6.4 \mathrm{kpc})$. The bar is embedded within a faint more circular outer disk that is visible out to a radius of $230^{\prime \prime}(17.2 \mathrm{kpc})$. The bright inner arms of the grand-design spirals lie on the leading edge of the stellar bar, delineating SF. [See the electronic edition of the Journal for a color version of this figure.] 
TABLE 1

AdOPTED PARAMETERS FOR NGC 5248

\begin{tabular}{lcc}
\hline \hline \multicolumn{1}{c}{ Parameter } & Value & Reference \\
\hline Hubble type $\ldots \ldots \ldots \ldots \ldots \ldots$. & SAB(rs)bc & 1,2 \\
Nuclear type $\ldots \ldots \ldots \ldots \ldots \ldots$ & H II & 3,4 \\
$D_{25}(\operatorname{arcsec}) \ldots \ldots \ldots \ldots \ldots \ldots$. & 370 & 2 \\
Distance $D(\mathrm{Mpc}) \ldots \ldots \ldots$. & 15.3 & 2 \\
Inclination $i(\mathrm{deg}) \ldots \ldots \ldots$. & $40 \pm 4$ & 5 \\
Line of nodes $(\mathrm{deg}) \ldots \ldots \ldots$ & $105 \pm 2$ & 5 \\
R.A. $(\mathrm{B} 1950.0) \ldots \ldots \ldots \ldots \ldots$ & 133502.54 & 2 \\
Decl. $(\mathrm{B} 1950.0) \ldots \ldots \ldots \ldots$. & 090821.56 & 2 \\
$V_{\text {sys }}\left(\mathrm{km} \mathrm{s}^{-1}\right) \ldots \ldots \ldots \ldots \ldots \ldots$ & 1153 & 2 \\
\hline
\end{tabular}

Note.-Units of right ascension are hours, minutes, and seconds, and units of declination are degrees, arcminutes, and arcseconds.

ReFerences.- (1) Nearby Galaxies (NBG) Catalogue; Tully 1988. (2) RC3; de Vaucouleurs et al. 1991. (3) Ho, Filippenko, \& Sargent 1997. (4) Kennicutt et al. 1989. (5) Jogee et al. 2002.

disk, and therefore the outer region of the bar was mistaken for an inclined outer disk. The corotation radius $\left(r_{\mathrm{CR}}\right)$ of the bar lies at $\sim 115^{\prime \prime}(8.6 \mathrm{kpc})$, assuming the empirical relationship $r_{\mathrm{CR}}=(1.2 \pm 0.2) a_{\mathrm{bar}}$ (Athanassoula 1992). Table 1 summarizes the parameters adopted for NGC 5248 in this paper. The inner kiloparsec of the bar hosts a well-known ring of "hot spots" (e.g., Buta \& Crocker 1993; Kennicutt, Keel, \& Blaha 1989; Elmegreen et al. 1997) that has been resolved into bright $\mathrm{H}$ II regions and young super-star clusters (SSCs) by Hubble Space Telescope (HST) broadband, $\mathrm{H} \alpha$, and $\mathrm{Pa} \alpha$ images (Maoz et al. 2001). Inside this ring resides a second nuclear $\mathrm{H} \alpha$ ring with a radius of 1".25 (Laine et al. 2001; Maoz et al. 2001) and a nuclear dust spiral (Laine et al. $1999 \mathrm{~b})$ between $1^{\prime \prime}(75 \mathrm{pc})$ and $4^{\prime \prime}(300 \mathrm{pc})$.

High-resolution observations of molecular gas can provide important constraints on the dynamics and evolution of the central regions in disk galaxies. Not only does the molecular gas fuel the ongoing SF, but its morphology and kinematics can also be a powerful tracer of nonaxisymmetric features in the underlying galactic potential (e.g., Knapen et al. 1995a; Sakamoto et al. 1995; Jogee et al. 1999; Knapen et al. 2000). We present new high-resolution $(1 " .9 \times 1 "$.4 or $150 \times 100$ pc) Owens Valley Radio Observatory $(\mathrm{OVRO}) \mathrm{CO}(J=1-0)$ observations of NGC 5248 and compare the data with Fabry-Perot $\mathrm{H} \alpha$ observations (Laine et al. 2001), optical and NIR broadband images, archival HST images (Maoz et al. 2001), and theoretical models. The main sections in this paper are organized as follows. In $\S 2$ we outline the observations and data reduction. In $\S 3$ we describe the stellar, dust, and gaseous grand-design spirals in NGC 5248. In $\S 4$ we present the CO and $K$-band data, showing the striking molecular and young stellar spirals. We describe the $\mathrm{CO}$ kinematics and present the rotation curve of NGC 5248 in $\S 5$. In $\S 6$ we discuss evolution in the inner kiloparsec of NGC 5248, addressing in particular the feeding of the starburst ring with cold molecular fuel, the local SF properties based on different tracers, and the interplay of the SSCs with the interstellar medium. In $\S 7.1$ estimates of the number and locations of the ILRs in NGC 5248 are presented. To account for the observed properties, the data are compared to new revised theoretical models in $\S 7.2$. In $\S 8$ we discuss the implications of this work for ISM redistribution and galaxy evolution.

\section{OBSERVATIONS}

\subsection{CO Interferometric Observations}

The central $65^{\prime \prime}$ of NGC 5248 were observed in the redshifted CO $(J=1-0)$ transition at $114.83 \mathrm{GHz}$ with the OVRO millimeter-wave array (Padin et al. 1991) between 1999 March and 2001 February. The array consists of six $10.4 \mathrm{~m}$ telescopes with a primary half-power beamwidth of $65^{\prime \prime}$ at $115 \mathrm{GHz}$. The galaxy was observed in five array configurations that range from compact to ultrawide and include projected baselines from 12 to $483 \mathrm{~m}$. Data were obtained simultaneously with an analog continuum correlator of bandwidth $1 \mathrm{GHz}$ and a digital spectrometer setup that produced four independent modules that each have 32 channels with a velocity resolution of $5.2 \mathrm{~km} \mathrm{~s}^{-1}$. For our observations, the modules were partially overlapping and covered a total bandwidth of $240 \mathrm{MHz}\left(600 \mathrm{~km} \mathrm{~s}^{-1}\right)$ with 116 frequency channels. We corrected for temporal phase variations by interspersing integrations on the galaxy with observations of a phase calibrator, typically a quasar, every 20-25 minutes. Passbands were calibrated on the bright quasars 3C 273, 3C 84, and 3C 345. The absolute flux scale was determined from observations of Uranus, Neptune, and 3C 273 and has an accuracy of 20\%. The passband and flux calibration of the data were carried out using the Owens Valley millimeter array software (Scoville et al. 1993). We used the NRAO AIPS software to map the calibrated $u v$ data and to deconvolve the channel maps with the CLEAN algorithm and robust weighting as implemented in the AIPS task IMAGR. Primary beam correction was applied. The parameters of the channel maps are summarized in Table 2.

TABLE 2

Parameters of OVRO Channel Maps

\begin{tabular}{|c|c|}
\hline Parameter & Value \\
\hline \multicolumn{2}{|l|}{ Pointing center (B1950.0): } \\
\hline R.A. . & 133502.60 \\
\hline Decl... & 090822.0 \\
\hline Projected baselines $(\mathrm{m})$.. & $12-483$ \\
\hline Transition ........... & $\mathrm{CO}(1-0)$ at $115 \mathrm{GHz}$ \\
\hline Half-power beamwidth $(\operatorname{arcsec})$............. & 65 \\
\hline Spectral bandwidth $\left(\mathrm{MHz}, \mathrm{km} \mathrm{s}^{-1}\right) \ldots$ & 240,600 \\
\hline Spectral resolution $\left(\mathrm{km} \mathrm{s}^{-1}\right) \ldots \ldots \ldots \ldots \ldots$ & 5.2 \\
\hline Synthesized beam $(\operatorname{arcsec}, \mathrm{pc}) \ldots \ldots \ldots \ldots \ldots . . . . . .$. & $1.9 \times 1.4,140 \times 100$ \\
\hline P.A. (deg) & -80.91 \\
\hline Emission channels $\left(\mathrm{km} \mathrm{s}^{-1}\right) \ldots \ldots \ldots \ldots \ldots \ldots$ & $1012-1293$ \\
\hline Peak emission $\left(\mathrm{mJy}\right.$ beam $\left.^{-1}\right) \ldots \ldots \ldots \ldots \ldots \ldots$ & 210 \\
\hline Peak $T_{b}{ }^{\mathrm{a}}(\mathrm{K})$ & 6.9 \\
\hline Typical rms $\left(\mathrm{mJy}\right.$ beam $\left.^{-1}\right)$.. & 17 \\
\hline Flux in OVRO map $\left(\mathrm{Jy} \mathrm{km} \mathrm{s}^{-1}\right)$.. & 385 \\
\hline 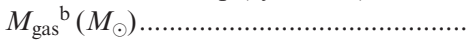 & $1.4 \times 10^{9}$ \\
\hline$F_{\mathrm{sd}}^{\mathrm{c}}(\%) .$. & 85 \\
\hline Single-dish flux $\left(\mathrm{Jy} \mathrm{km} \mathrm{s}^{-1}\right) \ldots$ & $450^{\mathrm{d}}$ \\
\hline
\end{tabular}

NoTE.-Units of right ascension are hours, minutes, and seconds, and units of declination are degrees, arcminutes, and arcseconds.

${ }^{a} T_{b}$ is the brightness temperature in Rayleigh-Jeans approximation.

${ }^{b} M_{\mathrm{gas}}$ is the mass of molecular gas including the contribution of helium, assuming a standard Galactic $\mathrm{CO}-\mathrm{H}_{2}$ conversion factor and a solar composition (see text).

${ }^{\mathrm{c}} F_{\mathrm{sd}}$ is the fraction of single-dish flux recovered.

d From Five College Radio Observatory observations (Young et al. 1995). 
Emission above the $3 \sigma$ level is detected in 55 channels in the velocity range of $1012-1293 \mathrm{~km} \mathrm{~s}^{-1}$. We combined the cleaned channel maps showing emission to make moment 0 , 1 , and 2 maps that represent the total intensity, the intensity-weighted velocity field, and the velocity dispersion field, respectively. Maps with different Briggs robustness parameters ranging from -7 to 4 were made to allow for different weighting schemes that compromise between resolution and signal-to-noise ratio. The maps discussed here contain a total flux of $385 \pm 20 \mathrm{Jy} \mathrm{km} \mathrm{s}^{-1}$ over the central $38^{\prime \prime}$, corresponding to $85 \%$ of the single-dish flux in the central $45^{\prime \prime}$ (Young et al. 1995).

\subsection{Near-Infrared and Optical Data}

We complement the $\mathrm{CO}$ observations with optical and NIR data, which are summarized in Table 3 . These include published archival HST broadband and $\mathrm{H} \alpha+\left[\mathrm{N} \mathrm{II}_{\mathrm{II}}\right.$ images, TAURUS Fabry-Perot $\mathrm{H} \alpha$ data (Laine et al. 2001), and $J-K$ color maps. We also obtained images of NGC 5248 through the $R, B$, and $K_{s}$ filters.

NGC 5248 was imaged through the Harris $R$-band filter for a total exposure time of 30 minutes using the Wide Field Camera on the $2.5 \mathrm{~m}$ Isaac Newton Telescope (INT) in La Palma in 2001 August. The data frames had a plate scale of $0 ! 33$ pixel $^{-1}$, a field of view of $11 ! 3 \times 22 ! 5$, and an average seeing of 1".8. Bias-subtracted and flat-fielded frames were obtained from the INT data reduction pipeline. Sky fringing at the $1 \%$ level is present, an effect known for the thinned EEV chip CCD4. We used the IRAF package for fixing bad pixels, cleaning cosmic rays, and convolving the frames to a common seeing before combining them into a final image. After masking out stars and faint background galaxies in the outer parts of the final image, isophotal analysis of the $R$-band light was performed with the "isophote" package in IRAF. Details are presented in Paper I.

A $B$-band image of NGC 5248, taken with the Prime Focus Camera on the $2.5 \mathrm{~m}$ Isaac Newton Telescope with a total exposure time of 20 minutes, was retrieved from the Isaac Newton Group archive. The image has 0"589 pixels and a field of view of $11^{\prime}$. We used the IRAF package in the standard way for data reduction. NGC 5248 was also imaged through the $K$-short $\left(K_{s}\right)$ filter for a total on-source exposure time of 12 minutes using the INGRID camera on the $4.2 \mathrm{~m}$ William Herschel Telescope. Seeing was 0".8. Onsource frames were interspersed with off-source frames pointed on a nearby blank area of the sky for reliable sky subtraction. The final reduced images have 0 ".24 pixels and a 5!1 field of view. The data acquisition and reduction are described in more detail in J. H. Knapen et al. (2002, in preparation).

\section{THE GRAND-DESIGN SPIRALS IN NGC 5248}

NGC 5248 exhibits spectacular grand-design spiral structure in all disk components, namely, gas, dust, and young stars. Spiral arms exist from $\sim 10 \mathrm{kpc}$ to $\sim 100 \mathrm{pc}$ and cover more than $360^{\circ}$ in azimuth, as illustrated by Figures 1-7. The $R$-band (Fig. 1) and $B$-band (Fig. 2) images show two bright, relatively symmetric spirals that are particularly prominent on the leading edge of the large-scale stellar bar

TABLE 3

Summary of Data on NGC 5248 Presented in This Paper

\begin{tabular}{|c|c|c|c|c|}
\hline $\begin{array}{l}\text { Data } \\
\text { (1) }\end{array}$ & $\begin{array}{c}\text { Instrument/Telescope } \\
\text { (2) }\end{array}$ & $\begin{array}{l}t_{\exp } \\
(3)\end{array}$ & $\begin{array}{c}\text { Field of View } \\
\text { (4) }\end{array}$ & $\begin{array}{l}\text { Source } \\
\text { (5) }\end{array}$ \\
\hline $\mathrm{CO}(1-0) \ldots$ & OVRO mm array & $8 \times 6^{\mathrm{a}}$ & $65 \times 65^{\mathrm{b}}$ & 1 \\
\hline $\mathrm{H} \alpha+\left[\mathrm{N}_{\mathrm{II}}\right] \ldots \ldots \ldots \ldots . . . .$. & WFPC $2 / H S T$ & $4800^{c}$ & $35^{\mathrm{b}}$ & 2 \\
\hline 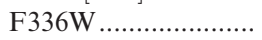 & WFPC $2 / H S T$ & $2300^{\mathrm{c}}$ & $35^{\mathrm{b}}$ & 2 \\
\hline 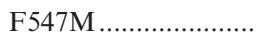 & WFPC $2 / H S T$ & $900^{\mathrm{c}}$ & $35^{\mathrm{b}}$ & 2 \\
\hline 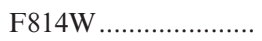 & WFPC $2 / H S T$ & $1100^{\mathrm{c}}$ & $35^{\mathrm{b}}$ & 2 \\
\hline Harris $R \ldots \ldots \ldots \ldots \ldots \ldots$ & WFC/INT $2.5 \mathrm{~m}$ & $30^{\mathrm{d}}$ & $11.3 \times 22.5^{\mathrm{e}}$ & 1,3 \\
\hline Harris $B \ldots \ldots \ldots \ldots \ldots \ldots$ & $\mathrm{PFC} / \mathrm{INT} 2.5 \mathrm{~m}$ & $20^{\mathrm{d}}$ & $11.0 \times 11.0^{\mathrm{e}}$ & 4 \\
\hline 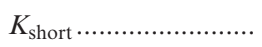 & INGRID/WHT $4.2 \mathrm{~m}$ & $12^{\mathrm{d}}$ & $5.1 \times 5.3^{\mathrm{e}}$ & 5 \\
\hline Fabry-Perot $\mathrm{H} \alpha \ldots \ldots$. & TAURUS II/WHT $4.2 \mathrm{~m}$ & $55 \times 110^{\mathrm{c}}$ & $1.4 \times 1.4^{\mathrm{e}}$ & 6 \\
\hline$J-K$ map $\ldots \ldots \ldots \ldots \ldots \ldots$ & MONICA-AO/CFHT $3.6 \mathrm{~m}$ & $180^{\mathrm{c}}$ & $12.4 \times 12.4^{\mathrm{b}}$ & 7 \\
\hline$J-K$ map $\ldots \ldots \ldots \ldots \ldots \ldots$ & MONICA/CFHT $3.6 \mathrm{~m}$ & $300^{\mathrm{c}}$ & $1.0 \times 1.0^{\mathrm{e}}$ & 7 \\
\hline
\end{tabular}

Note.-Col. (1): Description of the data. We quote the transition for the interferometric data and the filter for the images. Col. (2): Instrument and telescope. Abbreviations: OVRO = Owens Valley Radio Observatory; WFPC2/HST = Wide Field Planetary Camera 2 on the Hubble Space Telescope; WFC/INT = Wide Field Camera on the Isaac Newton Telescope at La Palma; PFC/ INT = Prime Focus Camera on the Isaac Newton Telescope at La Palma; INGRID/WHT = INGRID camera on the William Herschel Telescope at La Palma; TAURUS II/WHT = TAURUS II instrument in Fabry Perot mode on the William Herschel Telescope at La Palma; MONICA-AO/ CFHT $=$ combination of Montreal NIR camera MONICA with the adaptive optics system PUEO on the Canada-France-Hawaii Telescope at Mauna Kea. Col. (3): On-source exposure time. Col. (4): Field of view. For OVRO, the primary half-power beamwidth at $115 \mathrm{GHz}$ is quoted. Col. (5): Source of data.

a In hours.

b In arcseconds.

${ }^{\mathrm{c}}$ In seconds.

d In minutes.

e In arcminutes.

REFERENCES.- (1) This paper. (2) Archival HST images first published by Maoz et al. 2001. (3) Jogee et al. 2002. (4) Isaac Newton Group archive. (5) J. H. Knapen et al. 2002, in preparation. (6) Laine et al. 2001. (7) Laine et al. 1999b. 


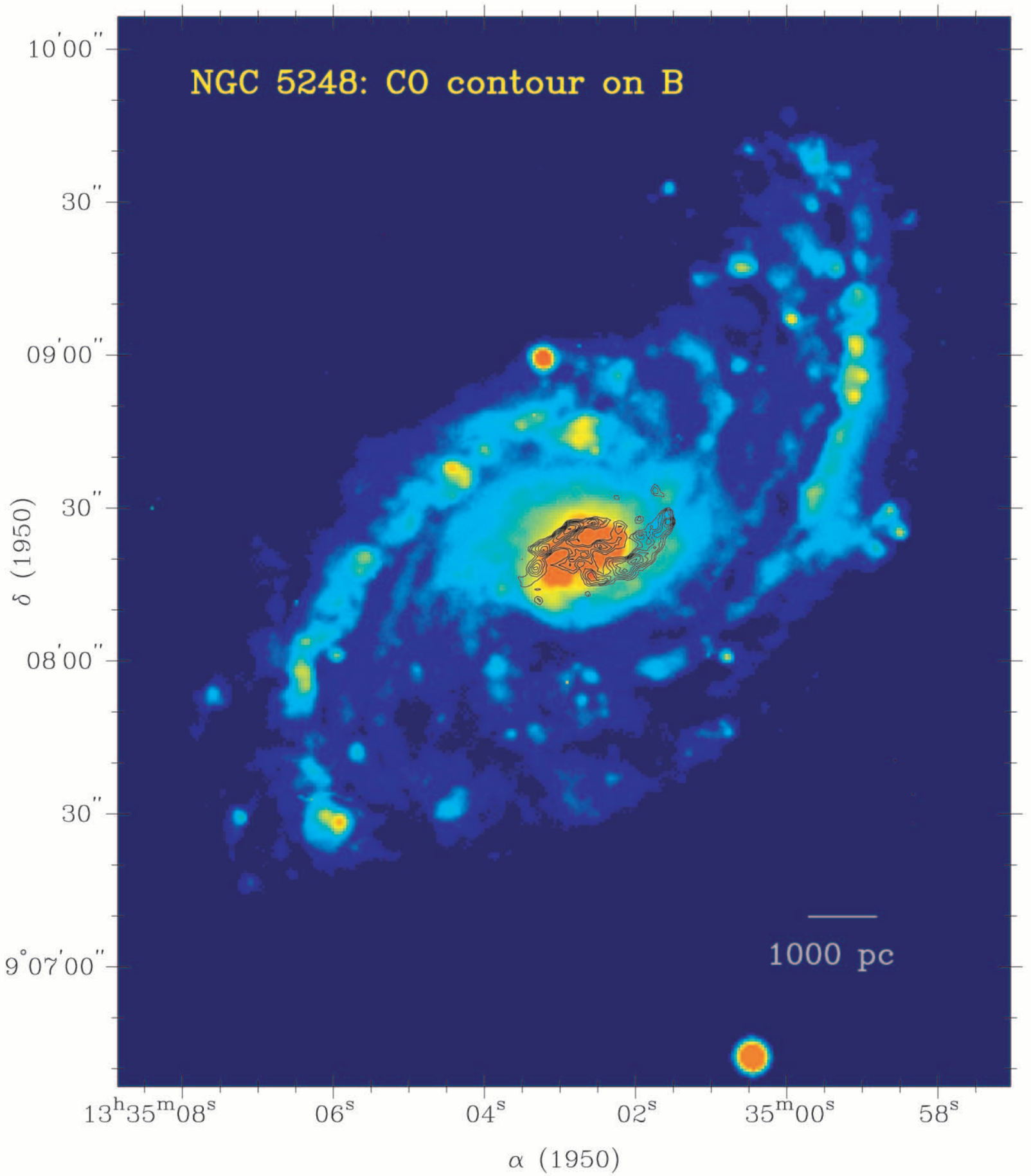

FIG. 2.-Optical grand-design spirals. The CO (1-0) intensity map (contours) of the central $40^{\prime \prime}$ is overlaid on a $B$-band image. Two bright stellar spiral arms, lined with young star-forming regions, are conspicuous between $\sim 30^{\prime \prime}$ and $90^{\prime \prime}$. The arms host prominent dust lanes on their inner (concave) sides out to at least $70^{\prime \prime}$. The two massive trailing CO spiral arms almost connect to the $B$-band arms and can be followed from $20^{\prime \prime}$ down to $5^{\prime \prime}$.

between $30^{\prime \prime}$ and $90^{\prime \prime}$. These stellar spiral arms exhibit dust lanes on their inner (concave) side out to at least $70^{\prime \prime}$ (Fig. 2 and Paper I), as expected inside the corotation resonance (CR). The offset between the dust and young stars is consistent with the view that shocks, seen as dust lanes along the leading edges of a moderately strong bar, compress gas to form massive young stars. SF in the bar of NGC 5248 is exceptionally strong, even in the NIR (Paper I). Patchy $K$ band spirals (Fig. 3) accompany the $B$-band spirals on the leading edge of the bar, and peaks in $K$-band emission coin- cide with $\mathrm{H}$ II regions in the large-scale $\mathrm{H} \alpha$ image. The strong SF along the moderately strong bar in NGC 5248 is consistent with the current understanding of gas flows and SF in bars. Namely, in strong bars that exhibit almost straight offset dust lanes believed to trace strong shocks, the strong shear in the postshock flow can inhibit SF (e.g., Athanassoula 1992). However, in weaker bars with curved offset dust lanes, the weaker shocks and shear can induce SF rather than inhibit it. For instance, the collapse of gas cores to form stars can be induced by weak shocks with speeds of 


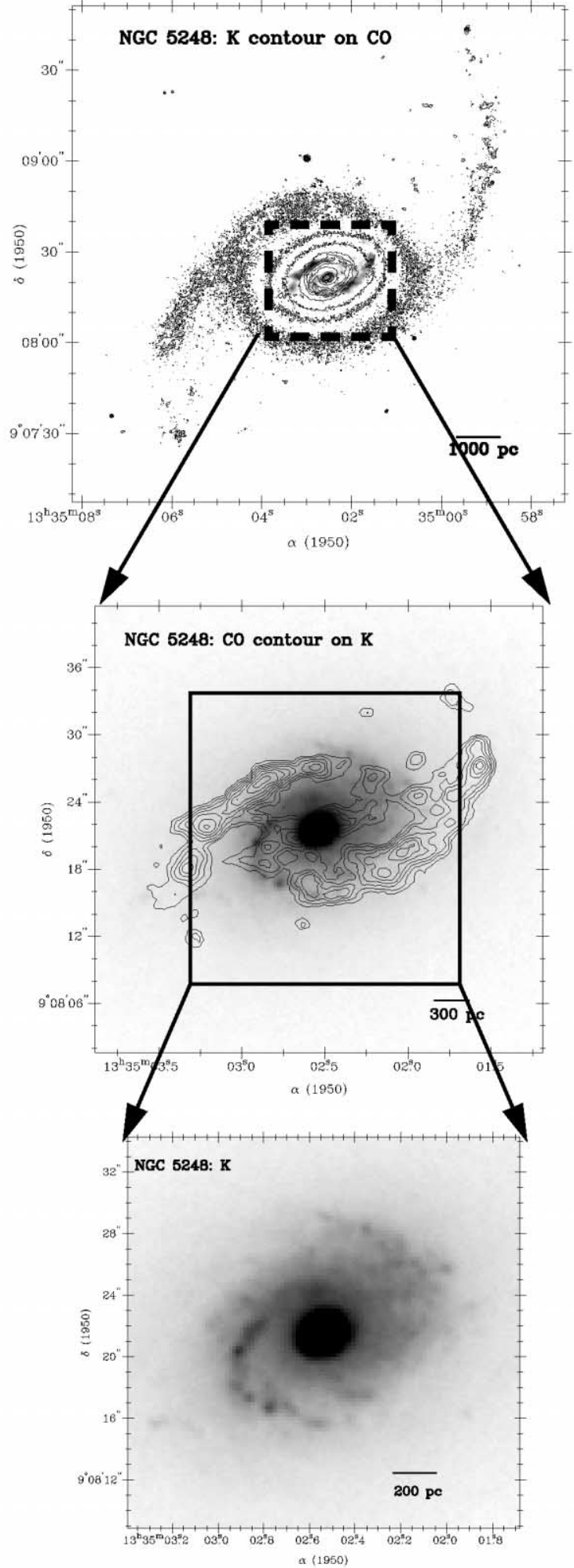

FIG. 3.-NIR morphology. Top: CO (1-0) intensity map (gray scale) of the central $40^{\prime \prime}$ overlaid on a $K_{s}$ image (contours) of the central 2!7. The NIR image shows a weakly oval feature within the central $22^{\prime \prime}(1.6 \mathrm{kpc})$ radius and prominent $K$-band spirals that can be easily traced from $85^{\prime \prime}$ down to $\sim 26^{\prime \prime}$ where they cross the bar major axis. The region inside the box is enlarged below. Middle: $\mathrm{CO}(1-0)$ intensity (contours) overlaid on the $K_{\mathrm{s}}$ image (gray scale) of the central $40^{\prime \prime}$. Bottom: $K_{\mathrm{s}}$ image (gray scale) in the central $25^{\prime \prime}$. After the outer $K$-band spirals cross the bar major axis, only very faint $K$-band emission is present between $26^{\prime \prime}$ and $9^{\prime \prime}$. At $\sim 8^{\prime \prime}$, two bright inner $K$-band spirals connect to the $\mathrm{CO}$ arms, cross the starburst ring while delineating the SSCs (see Figs. 6, 7, and 8), and continue down to $\sim 3^{\prime \prime}$ order 20-30 km s${ }^{-1}$ (e.g., Vanhala \& Cameron 1998). M100 (e.g., Elmegreen, Seiden, \& Elmegreen 1989; Knapen \& Beckman 1996), NGC 2903 (e.g., Sheth 2001), NGC 4254, and NGC 4303 (e.g., Koopmann 1997) are but a few examples of systems with significant SF along a weak bar. While in many strongly barred galaxies optical spiral arms are prominent outside the bar but not within it, NGC 5248 illustrates how intense SF along a fairly weak bar can lead to conspicuous open spiral arms within the bar itself.

The $R$-band spirals cross the bar major axis around $95^{\prime \prime}$ where the bar ends. This radius likely denotes the location of the ultraharmonic resonance where large-scale bars tend to end as a result of the onset of chaos and orbital instabilities. Secondary faint spirals stem off the bar and become particularly prominent from $115^{\prime \prime}$ to $150^{\prime \prime}$. These may be related to secondary compression zones that arise near CR (e.g., Buta 1984). Between $150^{\prime \prime}$ and $230^{\prime \prime}$, very faint spirals extend away from the bar end and cover $90^{\circ}-100^{\circ}$ in azimuth (Fig. 1) in the faint outer disk. The outer Lindblad resonance (OLR), where spiral arms are expected to end, is probably around $230^{\prime \prime}$.

The spiral structure in NGC 5248 not only extends out to large radii but also continues toward the central regions of the bar in three components, namely, gas, dust, and young stars. The $R$-band (Fig. 1), $B$-band (Fig. 2), $K$-band (Fig. 3), and $I-K$ (Fig. $4 a$ ) spirals can be readily followed from $70^{\prime \prime}$ to $26^{\prime \prime}$ where they cross the bar major axis. Interior to this point, the continuity in spiral structure is especially noticeable in the dust spirals revealed by the $I-K$ (Fig. $4 a$ ) and $J-K$ (Fig. $4 b$; see also Pérez-Ramírez et al. 2000) color maps. The dust spirals appear continuous from $70^{\prime \prime}$ to $5^{\prime \prime}$ and cover nearly another $180^{\circ}$ in azimuth between $26^{\prime \prime}$ and $5^{\prime \prime}$ before they join a circumnuclear starburst ring. The $\mathrm{CO}$ (1-0) maps described in $\S 4$ show that the dust spirals are associated with two massive molecular spiral arms that cover at least $180^{\circ}$ in azimuth between $20^{\prime \prime}$ and $5^{\prime \prime}$ (Fig. 5) and then connect to the starburst ring of $\mathrm{H}$ II regions (Fig. 6) and UV bright SSCs (Fig. 7). It is not entirely clear if the $K$ band spirals extend continuously from $70^{\prime \prime}$ to $5^{\prime \prime}$ as do the dust spirals: faint patches of $K$-band emission can be seen along the western molecular spiral between $19^{\prime \prime}$ and $12^{\prime \prime}$, and at $\sim 8^{\prime \prime}$ two striking bright inner $K$-band spirals (Fig. 8 ) connect to the molecular spirals. The narrow southern $K$-band spiral is particularly conspicuous. The $K$-band spirals continue from $600 \mathrm{pc}$ to $\sim 3^{\prime \prime}(225 \mathrm{pc})$ and delineate the UV bright SSCs as they cross the starburst ring (Figs. 7 and 8). Interior to the starburst ring an adaptive optics $J-K$ color map reveals a grand-design nuclear dust spiral (Fig. 9) that can be traced from $\sim 4^{\prime \prime}$ (300 pc) down to about $\sim 1^{\prime \prime}$ (75 pc; Laine et al. 1999b). This dust spiral appears to bridge the $K$ band spirals and the second nuclear $\mathrm{H} \alpha$ ring of radius $95 \mathrm{pc}$ where double peaks of $\mathrm{CO}$ emission can be seen $(\S 4)$. To account for the observed morphology, the data will be compared to theoretical models in $\S 7$, after a closer look at the molecular gas and SF properties in $\S \S 4-6$.

\section{THE CIRCUMNUCLEAR CO AND $K$-BAND MORPHOLOGY}

The OVRO CO (1-0) total intensity map (Fig. 4) has a significantly better resolution $(1 " .9 \times 1$." 4 or $140 \times 100 \mathrm{pc})$ than the previously published $(4 " .4 \times 3$ ".7 or $330 \times 280 \mathrm{pc})$ Nobeyama Millimeter Array (NMA) map (Sakamoto et al. 1999). This high-resolution map emphasizes the strongest 

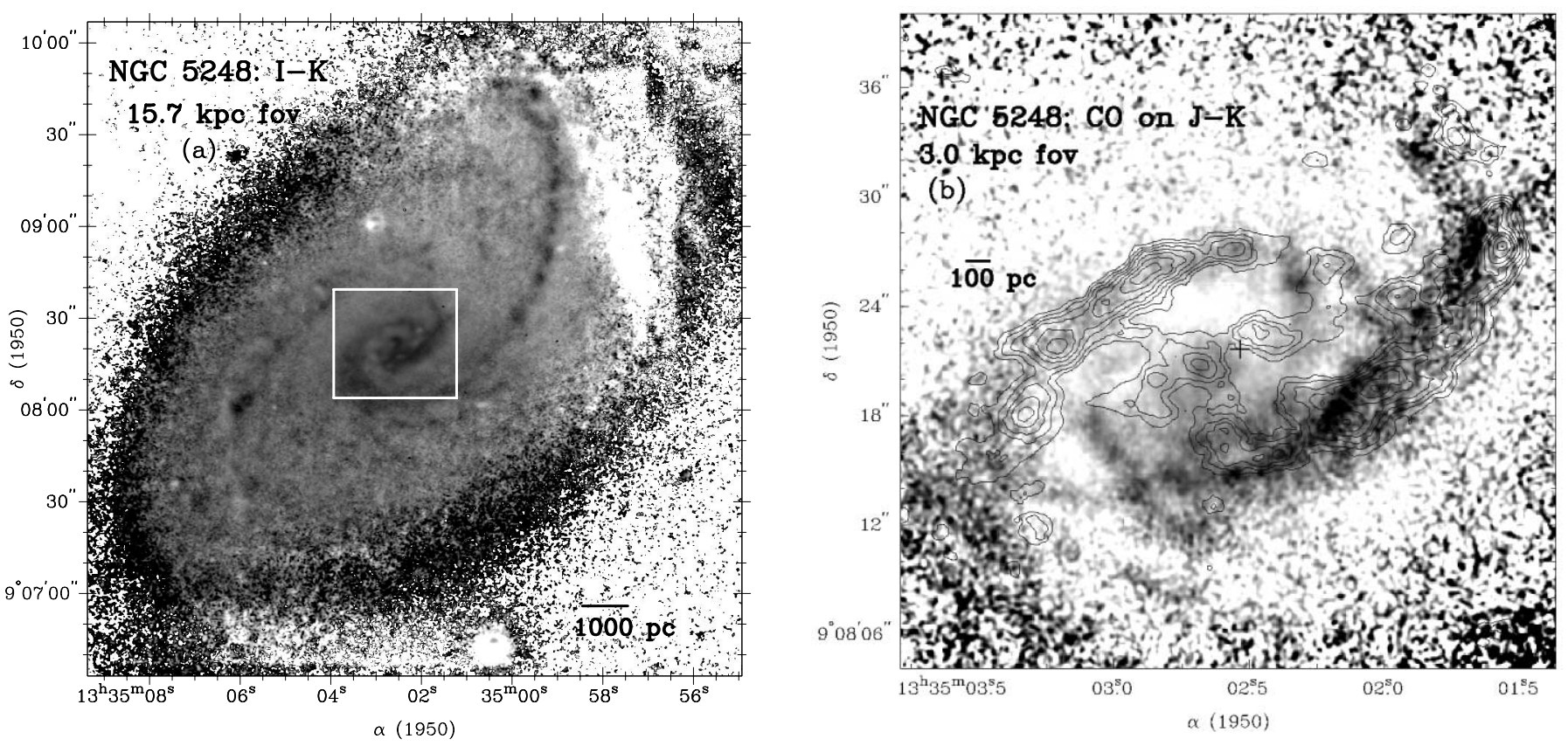

FIG. 4.-Grand-design dust spiral. (a) 2".4 resolution $I-K$ image showing a grand-design dust spiral that is continuous from at least $70^{\prime \prime}$ (5.2 kpc) down to $5^{\prime \prime}$ ( $375 \mathrm{pc}$ ). The region inside the white box is shown in (b). (b) CO (1-0) intensity map (contours) overlaid on a 1 ." 5 resolution $J-K$ color map (gray scale) of the central $3.0 \mathrm{kpc}$. The dust spiral follows the CO spiral arms between $20^{\prime \prime}$ and $5^{\prime \prime}$. [See the electronic edition of the Journal for a color version of Fig. $4 b$.]

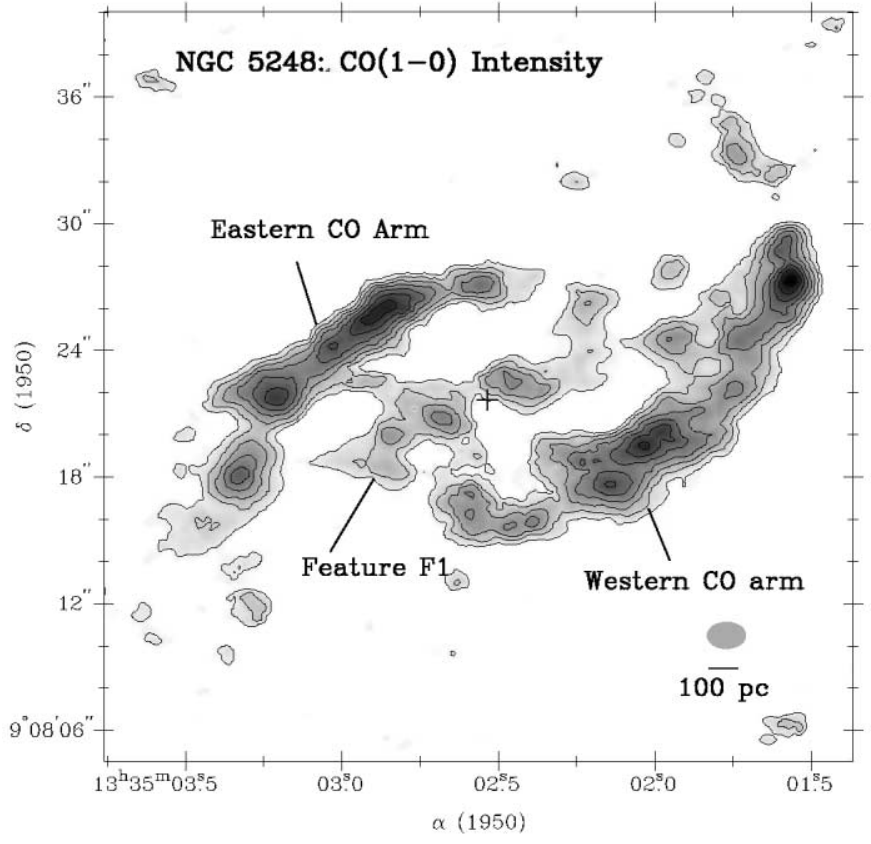

FIG. 5.-Molecular gas distribution in the inner kiloparsec. The CO (1$0)$ intensity map of the central $40^{\prime \prime}$ is shown. The 1 ". $9 \times 1$ ". $4(140 \times 100 \mathrm{pc})$ synthesized beam is marked. Contour levels are $20 \%, 35 \%, 45 \%, 55 \%, 65 \%$, $75 \%, 85 \%, 95 \%$, and $100 \%$ of the peak flux density $810 \mathrm{Jy} \mathrm{beam}^{-1} \mathrm{~km} \mathrm{~s}^{-1}$ and show regions of bright emission. The cross marks the peak of the HST $I$-band $(\mathrm{F} 814 \mathrm{~W})$ image. Two massive trailing $\mathrm{CO}$ spiral arms are seen from a radius of $20^{\prime \prime}$ to $5^{\prime \prime}$. The arms are resolved into clumps with sizes $\left(2^{\prime \prime}-4^{\prime \prime}\right.$, or $150-300 \mathrm{pc}$ ) and masses (several times $10^{6}$ to $10^{7} M_{\odot}$ ) comparable to giant molecular associations. Farther in resides a double-peaked $\mathrm{CO}$ feature surrounded by fainter emission. [See the electronic edition of the Journal for a color version of this figure.] features and may miss some of the fainter diffuse emission. Several striking features can be identified. Two relatively bisymmetric trailing $\mathrm{CO}$ spiral arms can be followed from a radius of $20^{\prime \prime}(1.5 \mathrm{kpc})$ to $5^{\prime \prime}(375 \mathrm{pc})$. The arms, also visible in the NMA map, are now resolved into multiple clumps with sizes of $2^{\prime \prime}-4^{\prime \prime}(150-300 \mathrm{pc})$ and masses in the range of several times $10^{6}-10^{7} M_{\odot}$, comparable to giant molecular associations (e.g., Vogel, Kulkarni, \& Scoville 1988). The $\mathrm{CO}$ arms connect to the circumnuclear starburst ring of H II regions (Fig. 6) and SSCs (Fig. 7) at a radius of 5". Inside this ring resides a double-peaked $\mathrm{CO}$ feature labeled $\mathrm{F} 1$ in Figure 5. Its two CO peaks lie partially on the inner nuclear $\mathrm{H} \alpha$ ring of radius 1".25 (95 pc) and are crossed by the arms of the grand-design nuclear dust spiral (Fig. 9; Laine et al. 1999b). Fainter CO emission is present in the starburst ring, northwest and southeast of the $\mathrm{CO}$ peaks. It is unclear if the apparent bridges between the faint $\mathrm{CO}$ emission and the two massive $\mathrm{CO}$ arms or the $\mathrm{CO}$ feature $\mathrm{F} 1$ are real or simply the result of beam smearing.

The massive $\mathrm{CO}$ arms are associated with prominent dust spirals (Fig. 4b) delineating shocks. These dust spirals form part of a continuous grand-design spiral that can be followed from $70^{\prime \prime}$ to at least $5^{\prime \prime}$ and crosses the bar major axis at $\sim 26^{\prime \prime}$. The $K_{s}$ image of $0 . " 8$ resolution (Fig. 8) shows some faint patches of emission along the western $\mathrm{CO}$ arm between $12^{\prime \prime}$ and $19^{\prime \prime}$ and two bright inner spirals that connect to the $\mathrm{CO}$ arms around $\sim 8^{\prime \prime}$. The $K$-band arms cross the starburst ring while delineating the UV bright SSCs (Fig. 8). The association between the southern $K$-band arm and the SSCs labeled SC11, SC12, SC13, and SC14 in Figure 7 is particularly striking. The emission peaks in the $K$-band arms are likely to be dominated by very young supergiants, 8-10 Myr old, which are often present in young star-forming regions (e.g., Leitherer \& Heckman 1995). The presence of such a 


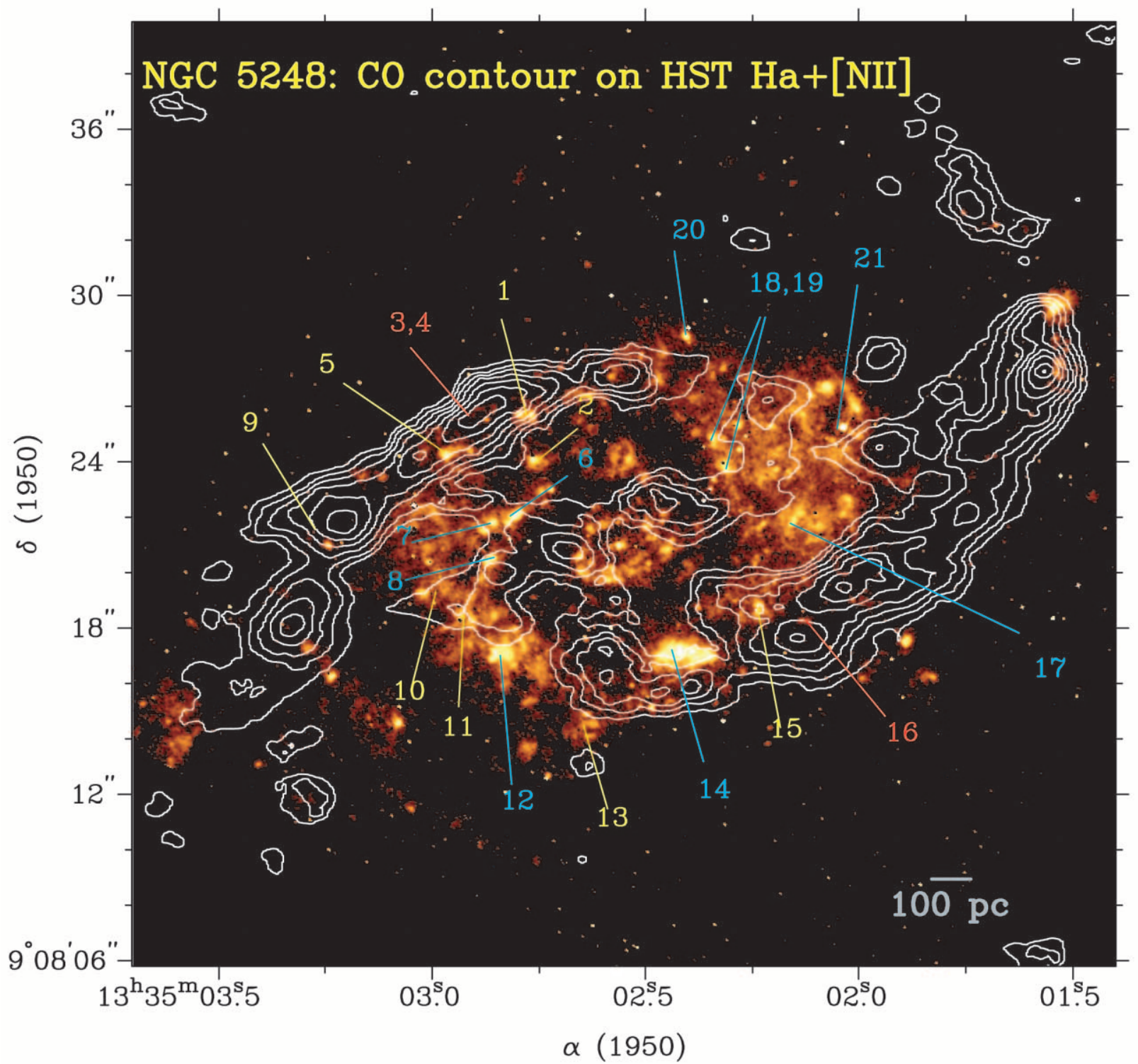

FIG. 6.-Distribution of $\mathrm{CO}$ and $\mathrm{H}$ II regions. The CO (1-0) intensity map (contours) is overlaid on the HST WFPC2 continuum-subtracted $\mathrm{H} \alpha+[\mathrm{N}$ II] image (gray scale; courtesy of Maoz et al. 2001). The two $\mathrm{CO}$ spiral arms connect to the circumnuclear starburst ring, which has a $5^{\prime \prime}$ radius and hosts $\mathrm{H}$ II regions and SSCs. The $\mathrm{H}$ II regions with identification numbers 3, 4, and 16 (labeled in red) have the largest extinction and show no optically visible stellar continuum sources although they have large $\mathrm{Pa} \alpha$ equivalent widths indicative of a young embedded stellar population a few Myr old. Interestingly, these complexes are situated in the CO arms and lie near bright CO peaks. Conversely, the complexes 8, 14, 21, 12, 6, 17, 19, 7, 18, and 20 (labeled in blue) have the lowest extinction, have typical ages of $10-40 \mathrm{Myr}$, and are associated with lower levels of $\mathrm{CO}$ emission. Inside the circumnuclear $\mathrm{H} \alpha$ ring resides a second $\mathrm{H} \alpha$ ring of radius 1 ". 25 , which is crossed by the two CO peaks of feature F1. Northwest and southeast of the CO peaks, fainter CO emission extends toward the $K$-band spirals in the circumnuclear ring.

young stellar population in the $K$-band arms is consistent with the study of Maoz et al. (2001), who estimate, from spectral synthesis, an age of 10-40 Myr for most of the bright SSCs. A young age for the $K$-band arms is also suggested by the narrow width of $0.6(110 \mathrm{pc})$ of the eastern $K$-band arm. For an assumed stellar velocity dispersion of $10 \mathrm{~km} \mathrm{~s}^{-1}$, this width suggests an age of order 10-20 Myr for the arms. Taken together, the morphology of the $\mathrm{CO}$ arms, dust spirals, young $K$-band arms, and SSCs suggests that SF in the starburst ring has been triggered by a bar-driven density wave.

The $K$-band arms are located just outside a very weak oval feature of radius $\sim 3^{\prime \prime}$ (Fig. 8). This feature has a deprojected ellipticity of $0.1-0.2$ at a position angle (P.A.) of $110^{\circ}-120^{\circ}$, slightly offset from the estimated P.A. of the line of nodes ( $105^{\circ}$; Fig. $10 a$ and Paper I). This feature could be a disklike component, a late-type bulge, or an unresolved nuclear bar. Interestingly, the grand-design nuclear dust spiral (Fig. 9) that continues from $3^{\prime \prime}$ to $1^{\prime \prime}$ appears to bridge the end of the $K$-band arms around $3^{\prime \prime}$ and the nuclear $\mathrm{H} \alpha$ ring of radius 1 1"25. The dust spiral also crosses the two CO peaks of feature F1. At the current resolution, it is not clear whether the CO peaks of F1 are part of a molecular spiral associated with the dust spiral or whether they are associated with a molecular ring coincident with the inner $\mathrm{H} \alpha$ ring.

We computed the mass of molecular hydrogen in our $\mathrm{CO}$ (1-0) maps using the relation (Kenney \& Young 1989; Sco- 


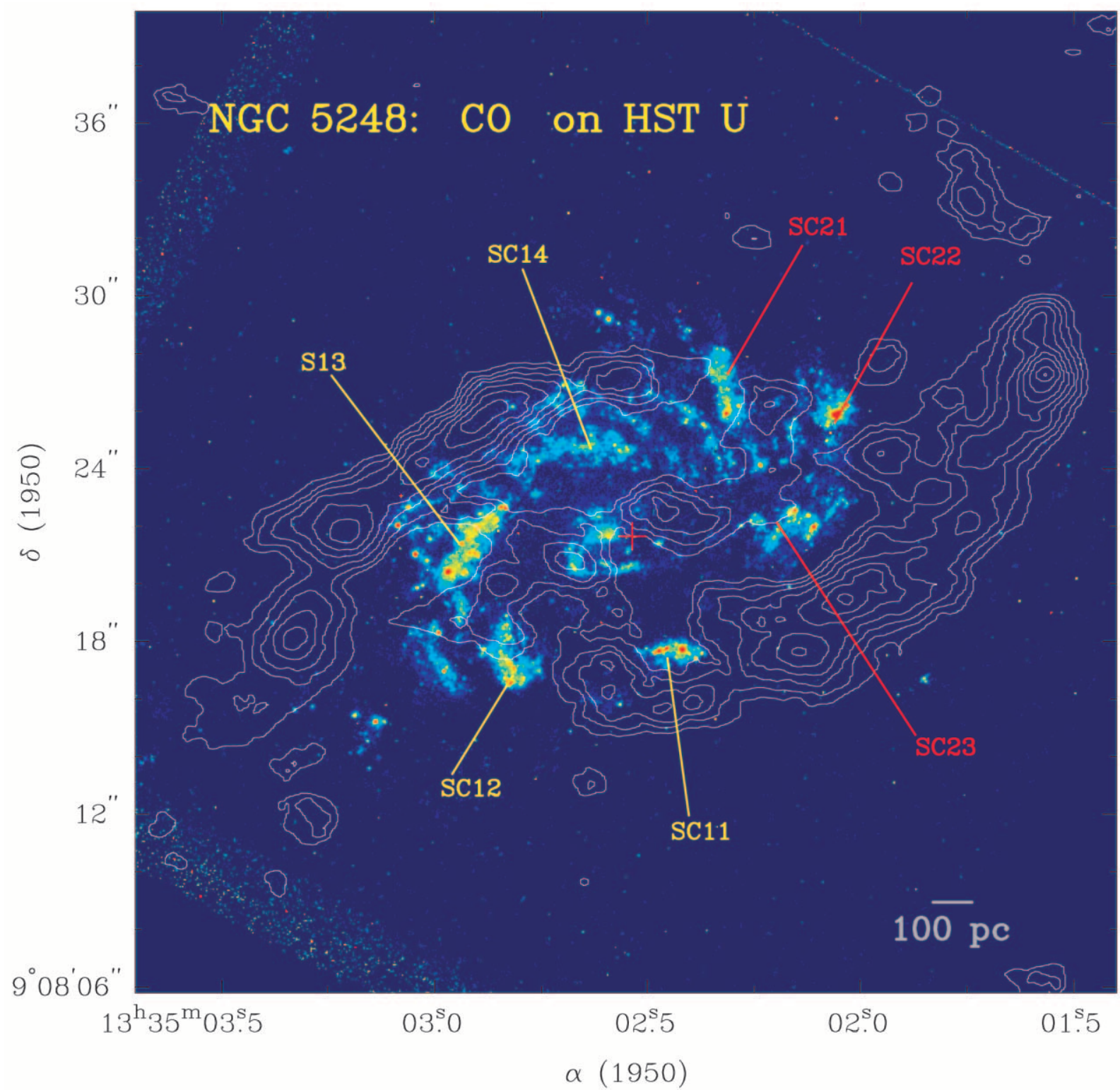

Fig. 7.-Distribution of CO and UV bright SSCs. The CO (1-0) intensity map (contours) is overlaid on the HST F336W image (gray scale). Peaks in CO and UV emission are generally anticorrelated. The stellar clusters labeled SC11, SC12, SC13, and SC14 lie along the narrow southern $K$-band spiral (Fig. 8), which connects to the western $\mathrm{CO}$ arm. The clusters SC21, SC22, and SC23 are more closely associated with the northern $K$-band spiral (Fig. 8), which connects to the eastern $\mathrm{CO}$ arm.

ville \& Sanders 1987)

$$
\frac{M_{\mathrm{H}_{2}}}{M_{\odot}}=1.1 \times 10^{4}\left(\frac{X}{2.8 \times 10^{20}}\right) D^{2} \int S_{\mathrm{CO}} d V
$$

where $D$ is the distance in Mpc, $\int S_{\mathrm{CO}} d V$ is the integrated line flux in units of $\mathrm{Jy} \mathrm{km} \mathrm{s}{ }^{-1}$, and $X$ is the $\mathrm{CO}-\mathrm{H}_{2}$ conversion factor. The value of $X$ in the inner Galaxy, namely, $2.8 \times 10^{20} \mathrm{H}_{2} \mathrm{~cm}^{-2} \mathrm{~K}\left(\mathrm{~km} \mathrm{~s}^{-1}\right)^{-1}$ (Bloemen et al. 1986), is generally referred to as the "standard" value. For an assumed standard value of the conversion factor, the two CO spiral arms host a total of $9.0 \times 10^{8} M_{\odot}$ of molecular hydrogen, while the $\mathrm{CO}$ feature F1 hosts $1.0 \times 10^{8} M_{\odot}$. Including the contribution of helium and heavier elements for a solar metallicity $(Z=0.02, X=0.72, Y=0.26)$ gives a molecular gas content of $1.2 \times 10^{9}$ and $1.4 \times 10^{8} M_{\odot}$, respectively. We estimate the enclosed dynamical mass using the circular rotation curve described in $\S 5$. In the central $20^{\prime \prime}(1.5 \mathrm{kpc})$ radius molecular gas makes up $11 \%$ of the enclosed dynamical mass $\left(1.2 \times 10^{10} M_{\odot}\right)$. Within the central $5^{\prime \prime}(375 \mathrm{pc})$ radius, the $\mathrm{CO}$ feature $\mathrm{F} 1$ makes up $7 \%$ of the dynamical mass $\left(2.1 \times 10^{9} M_{\odot}\right)$. Our mass estimates suffer from the usual caveats of assuming a standard value for the conversion factor. One may justifiably question this assumption since the conversion factor may depend on many parameters such as the dust column density, the ambient radiation field, the physical conditions in the gas, the optical thickness of the line, and the metallicity of the gas. These parameters could potentially show significant variations between different regions of galaxies. Furthermore, multiple-line studies and radiative transfer models (Wall \& Jaffe 1990; Wild et al. 1992; Helfer \& Blitz 1993; Aalto et al. 1995) have suggested lower values of $X$ in the centers of some starburst galaxies. However, several mitigating factors 


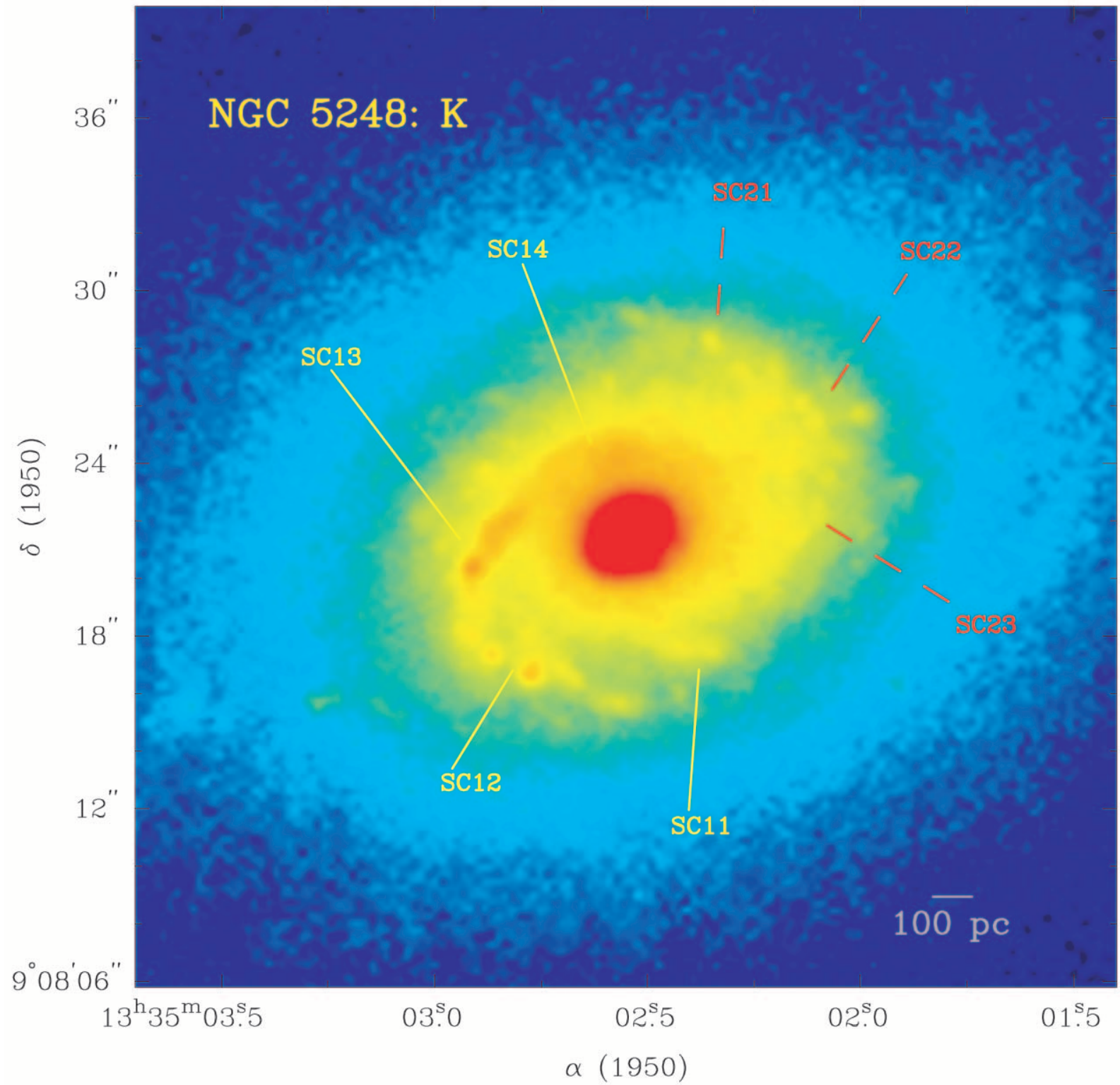

FIG. 8.- Inner $K$-band spirals delineating the SSCs. The $K_{s}$ image depicting the same region as Fig. 7 is shown. The positions of the UV bright SSCs in the $H S T$ UV (F336W) image (Fig. 7) are marked. Two $K$-band spirals extend from $3^{\prime \prime}$ to $8^{\prime \prime}$. The southern one is particularly clear and narrow, suggesting a young dynamical age. There is a striking spatial coincidence between emission peaks along the $K$-band spirals and the UV bright SSCs.

may help reduce deviations of the conversion factor from the standard value in the central region of NGC 5248. While circumnuclear star-forming regions very likely have higher temperatures and densities than Milky Way clouds, the effects of elevated temperatures and densities may partially cancel each other since $X$ depends on $\rho^{0.5} / T_{B}$ for clouds in virial equilibrium (e.g., Scoville \& Sanders 1987; Solomon et al. 1987), where $T_{B}$ is the $\mathrm{CO}$ brightness temperature averaged over the cloud and $\rho$ is the density. Furthermore, most of the CO emission in NGC 5248 is not associated with the ring of $\mathrm{H}$ II regions and SSCs ( $(6)$ where the radiation field from young stars might heat up the gas significantly. As far as metallicity is concerned, the circumnuclear region of an Sbc spiral such as NGC 5248 is likely to have a metallicity of at least solar, like most massive spirals (e.g., Vila-Costas \& Edmunds 1992). Over this regime of metallicity, the CO line is likely optically thick, and we expect at most a weak dependence of the $\mathrm{CO}-\mathrm{H}_{2}$ conversion factor on metallicity (e.g., Elmegreen 1989).

\section{THE MOLECULAR GAS KINEMATICS}

Figure $11 a$ shows the intensity-weighted $\mathrm{CO}$ velocity field of NGC 5248. The spectral resolution is $5.2 \mathrm{~km} \mathrm{~s}^{-1}$. The P.A. of the line of nodes $\left(105^{\circ}\right)$ determined from the outer disk in the $R$-band image (Paper I) is marked. The isovelocity contours deviate from a typical "spider diagram" that characterizes purely circular velocity fields. Noncircular streaming motions are especially visible in the $\mathrm{CO}$ arms where the isovelocity contours show significant curvatures. Noncircular motions are also apparent in the faint $\mathrm{CO}$ emission that lies around the two CO peaks of feature F1, near the ring of SF. In fact, the regions of noncircular motions 


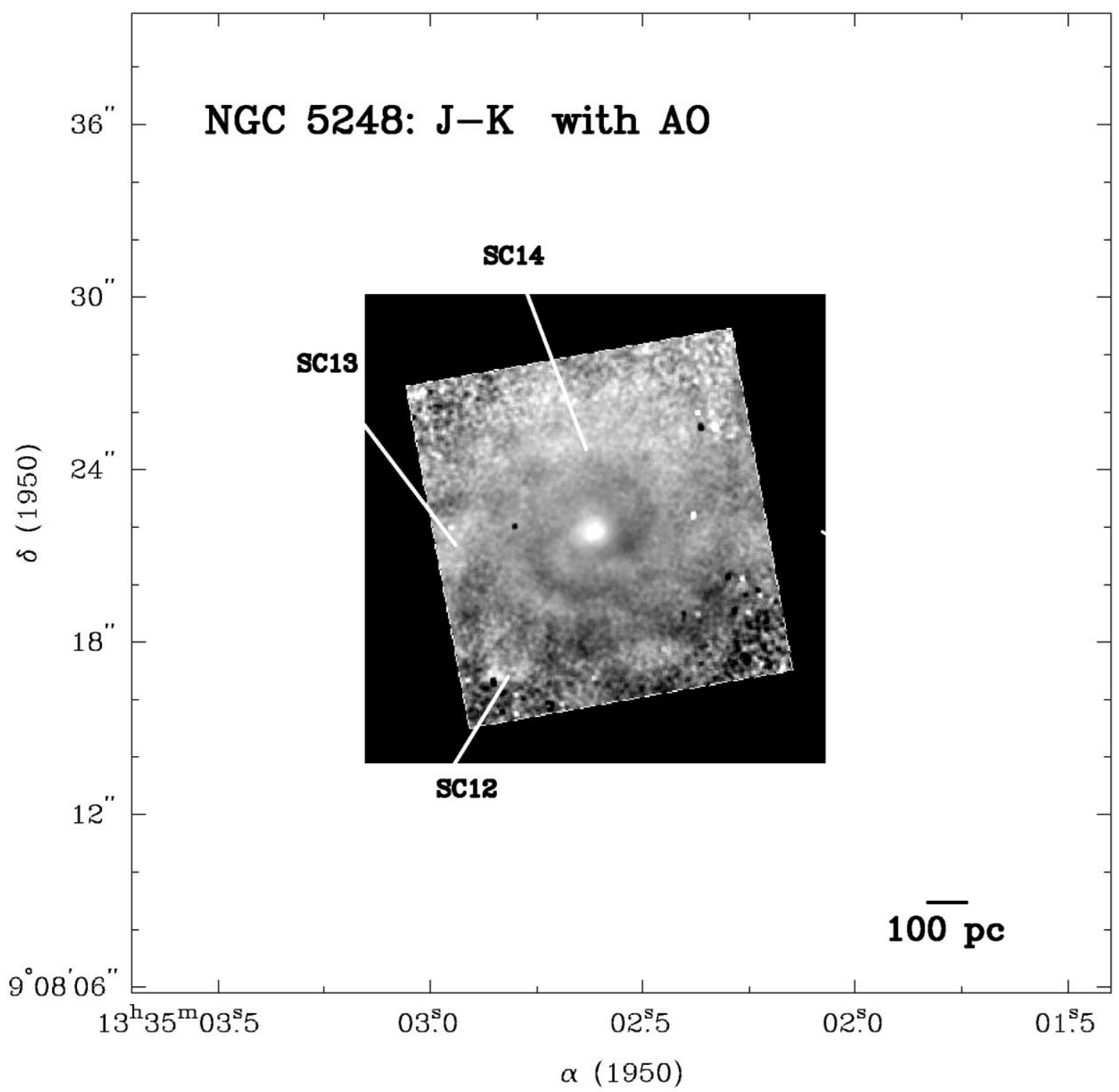

FIG. 9.- Nuclear grand-design dust spiral. The CFHT adaptive optics $J-K$ color index image (Laine et al. 1999b) of the core region of NGC 5248 shows the nuclear grand-design dust spiral in darker shades. The positions of three UV bright SSCs lying along the narrow western $K$-band arm, shown in Figs. 7 and 8 , are marked. Notice that the northern dust spiral appears to connect to this $K$-band arm around $3^{\prime \prime}$.

are intersected by the inner $K$-band spiral arms as they extend from $3^{\prime \prime}$ to $8^{\prime \prime}$, crossing the starburst ring.

At any general point, the line-of-sight velocity is a superposition of circular motions $\left(\Theta_{c}\right)$, azimuthal streaming motions $\left(\Theta_{s}\right)$, and radial streaming motions $(\Pi)$ in the plane, as well as velocities $(Z)$ out of the plane. It is particularly instructive to look at position-velocity cuts along the kinematic minor and major axes of the galaxy, where the components $\Theta_{s}$ and $\Pi$, respectively, tend to zero. The positionvelocity cut along the optical minor $\left(15^{\circ}\right)$ axis is shown in Figure $11 b$. At a distance of $6^{\prime \prime}-8^{\prime \prime}$ along the minor axis, where the position-velocity cut crosses the $\mathrm{CO}$ arms, the velocities to the northeast and southwest show, respectively, blueshifts and redshifts of $15-25 \mathrm{~km} \mathrm{~s}^{-1}$ with respect to the systemic velocity $\left(1153 \mathrm{~km} \mathrm{~s}^{-1}\right)$. These velocities correspond to local radial streaming motions ( $\Pi$ ) of $15-25 / \sin i \mathrm{~km} \mathrm{~s}^{-1}$ or $25-40 \mathrm{~km} \mathrm{~s}^{-1}$, if we assume that the optical minor axis is close to the kinematic minor axis and the out-of-plane $Z$-component of velocity is negligible. The radial streaming motions correspond to inflow if we assume that the northeast side of the galactic disk is the far side - an orientation justified at the end of this section. We caution, however, that local radial inflow motions do not directly translate into a net inflow rate (see $\S 6$ ).

The position-velocity cut (Fig. 11c) along the galaxy major axis or line of nodes $\left(105^{\circ}\right)$ first crosses the innermost part of the double-peaked $\mathrm{CO}$ feature $\mathrm{F} 1$ and then intersects the two $\mathrm{CO}$ arms. In the inner regions where we expect the rotation curve to be steeply rising, the position-velocity diagram shows a turnover velocity of $115 / \sin i \mathrm{~km} \mathrm{~s}^{-1}$ at a $7^{\prime \prime}$ radius. This corresponds to $179 \mathrm{~km} \mathrm{~s}^{-1}$ for a revised inclination of $40^{\circ}$ adopted from Paper I. Between $8^{\prime \prime}$ and $11^{\prime \prime}$ along the major axis, there is a dip in the mean $\mathrm{CO}$ velocity and an increase in the CO line width. This happens when the posi- 

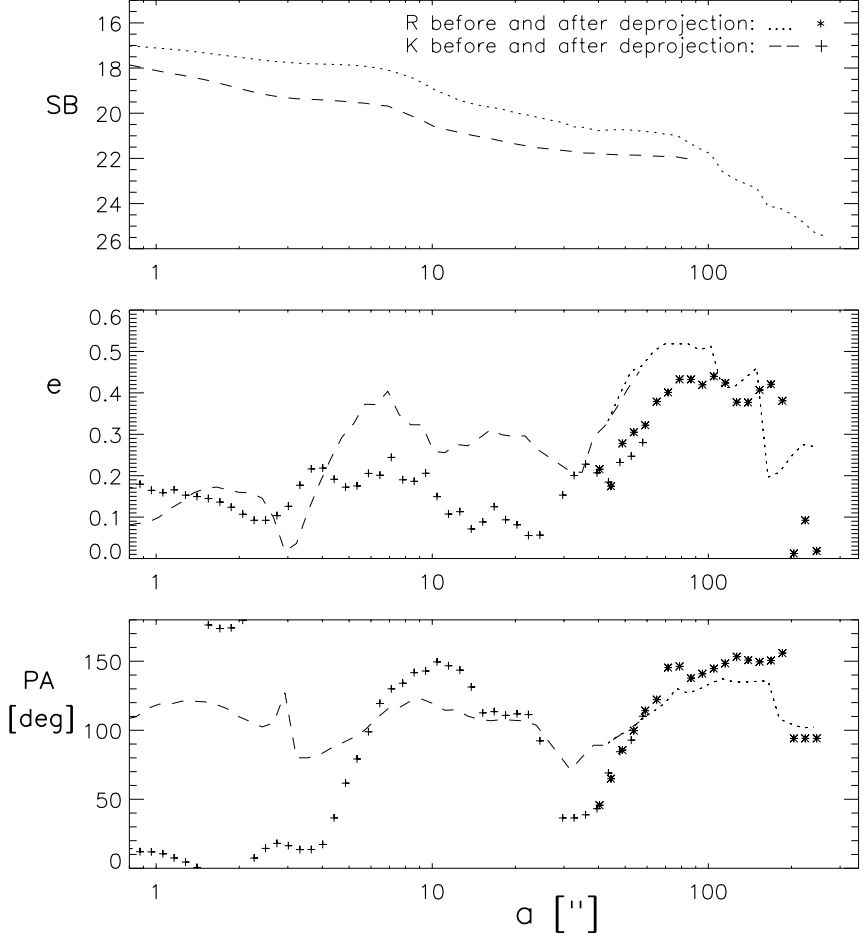

FIG. $10 a$

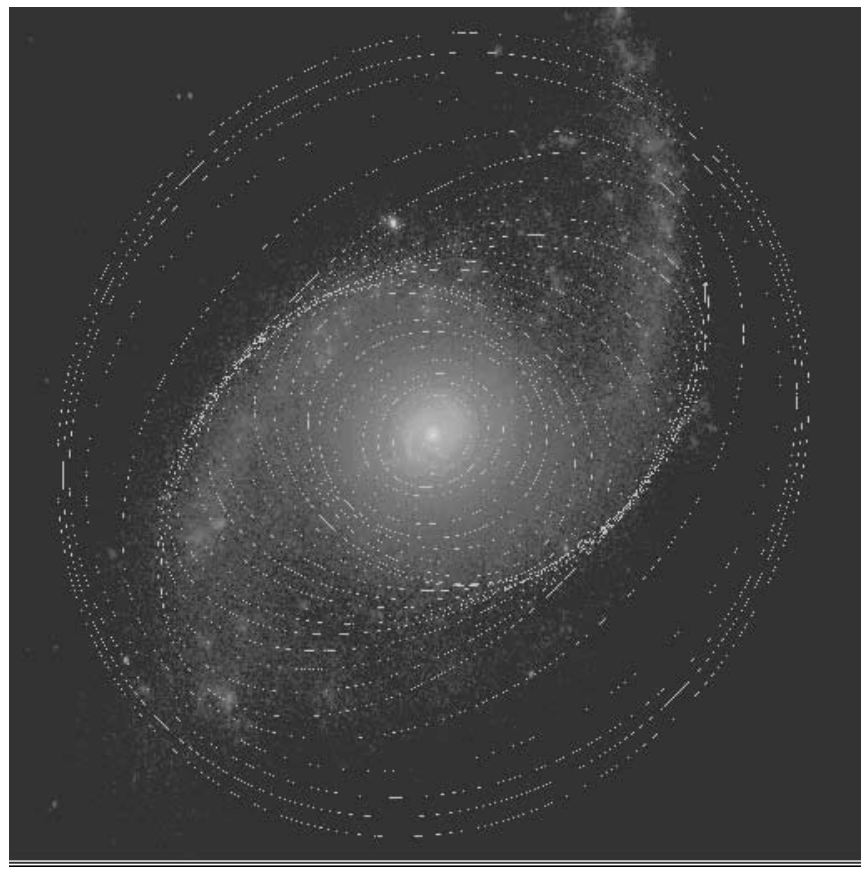

FIG. $10 b$

FIG. 10.-Isophotal analysis. (a) Radial profiles of surface brightness (SB), ellipticity (e), and P.A. of the $K_{s}$ and $R$-band light. The dotted and dashed lines refer to the values before deprojection. The symbols refer to deprojected values. The $K_{s} \mathrm{SB}$ has been scaled by 1.32 so that it can be plotted on the same scale as the $R$-band profile. In deprojected images, we adopt the convention that P.A. values are measured anticlockwise from "North." The gradual change in P.A. (isophotal twist) between $3^{\prime \prime}$ and $9^{\prime \prime}$ is caused by $K$-band arms. (b) Fitted isophotes to the deprojected $K_{s}$ image provide a guide to the dominant periodic stellar orbits present and the location of dynamical resonances. tion-velocity cut first intersects the $\mathrm{CO}$ arms (Fig. 11a). The curvatures in the isovelocity contours at that location suggest that the kinematic behavior in Figure $11 c$ results from noncircular azimuthal streaming motions. Farther out, from $12^{\prime \prime}$ to $18^{\prime \prime}$ along the major axis, the position-velocity cut crosses the two $\mathrm{CO}$ arms, and the line-of-sight velocity rises by about $40 \mathrm{~km} \mathrm{~s}^{-1}$. Again, the strong curvatures in the isovelocity contours (Fig. 11b) suggest that this rise is in large part due to noncircular streaming motions.

Fabry-Perot $\mathrm{H} \alpha$ observations of NGC 5248 with a spectral resolution of $20.0 \mathrm{~km} \mathrm{~s}^{-1}$ have been presented by Laine et al. (2001). They derive a best-fit model "circular" velocity in the central $10^{\prime \prime}$ by minimizing the residual velocity difference between the observed Fabry-Perot $\mathrm{H} \alpha$ velocity field and the model velocities. In the presence of strong noncircular motions, the best-fit circular velocity derived using this method is still contaminated by noncircular components. Their best-fit circular rotation curve derived assuming a line of nodes of $105^{\circ}$ and an inclination of $40^{\circ}$ is shown on Figure 12. The Fabry-Perot $\mathrm{H} \alpha$ circular velocity of $117 / \sin i$ $\mathrm{km} \mathrm{s}^{-1}$ at a $5^{\prime \prime}$ radius is consistent with the $\mathrm{CO}$ velocity of $115 / \sin i \mathrm{~km} \mathrm{~s}^{-1}$ at that location. Around $8^{\prime \prime}-10^{\prime \prime}$, the $\mathrm{H} \alpha$ velocity field shows residual noncircular motions of $30 \mathrm{~km}$ $\mathrm{s}^{-1}$ to the northeast and $15-20 \mathrm{~km} \mathrm{~s}^{-1}$ to the south and west. These noncircular motions occur near the intersection point of the $K$-band arms and $\mathrm{CO}$ arms with the starburst ring.

CO (1-0) observations with a lower resolution $\left(5^{\prime \prime}-6^{\prime \prime}\right)$ but a larger field of view $\left(2^{\prime}\right)$ have been carried out with the Berkeley Illinois Maryland Array (BIMA), as part of the Survey of Nearby Galaxies (SONG; Regan et al. 2001). The data (M. Das et al. 2002, in preparation) show molecular gas along the large-scale bar out to $90^{\prime \prime}$, as well as in the circumnuclear regions. A best-fit circular rotation curve derived from the BIMA data was kindly provided to us by M. Das prior to publication. It was derived with a technique of minimizing residuals, similar to the method applied by Laine et al. (2001) to the Fabry-Perot $\mathrm{H} \alpha$ data. It suffers, therefore, from the same caveats of possible contamination by noncircular motions. We used the high-resolution Fabry-Perot $\mathrm{H} \alpha$ and OVRO CO (1-0) circular rotation curve from 0 to $9^{\prime \prime}$ and the BIMA data of lower resolution from $13^{\prime \prime}$ to $80^{\prime \prime}$ to derive an overall rotation curve from the center to $80^{\prime \prime}$. This is shown in Figure 12, where values are already corrected for an assumed inclination of $40^{\circ}$. We did a cubic spline fit to interpolate between data points. The turnover velocity of $117 / \sin i$ or $182 \mathrm{~km} \mathrm{~s}^{-1}$ at a $7^{\prime \prime}$ radius is visible. Based on the above discussions of Figure $11 c$, we suspect that the dip in velocity from $8^{\prime \prime}$ to $11^{\prime \prime}$ and the $40 \mathrm{~km}$ $\mathrm{s}^{-1}$ rise in velocity from $10^{\prime \prime}$ to $20^{\prime \prime}$ are largely caused by noncircular motions.

Finally, we justify the assumption made earlier that the northeast side of the disk is the far side. The stars in the disk must be rotating in a clockwise sense if the large-scale stellar spiral arms are trailing, as is commonly the case with largescale spirals. We can also infer a clockwise sense of rotation for the stars from the following line of arguments. If we start on the bar major axis and move in a clockwise sense, we first cross the dust lanes on the leading edge of the bar, then the quasi-coincident $\mathrm{H} \alpha, K$-band, and $B$-band spirals (Paper I). This suggests that shocks, which we see as dust lanes along the leading edges of the bar, compress the gas to form massive young stars that move clockwise faster than the pattern speed and lead to the spirals made of young stars a little farther away. Such offsets between dust, gas, and stars have 


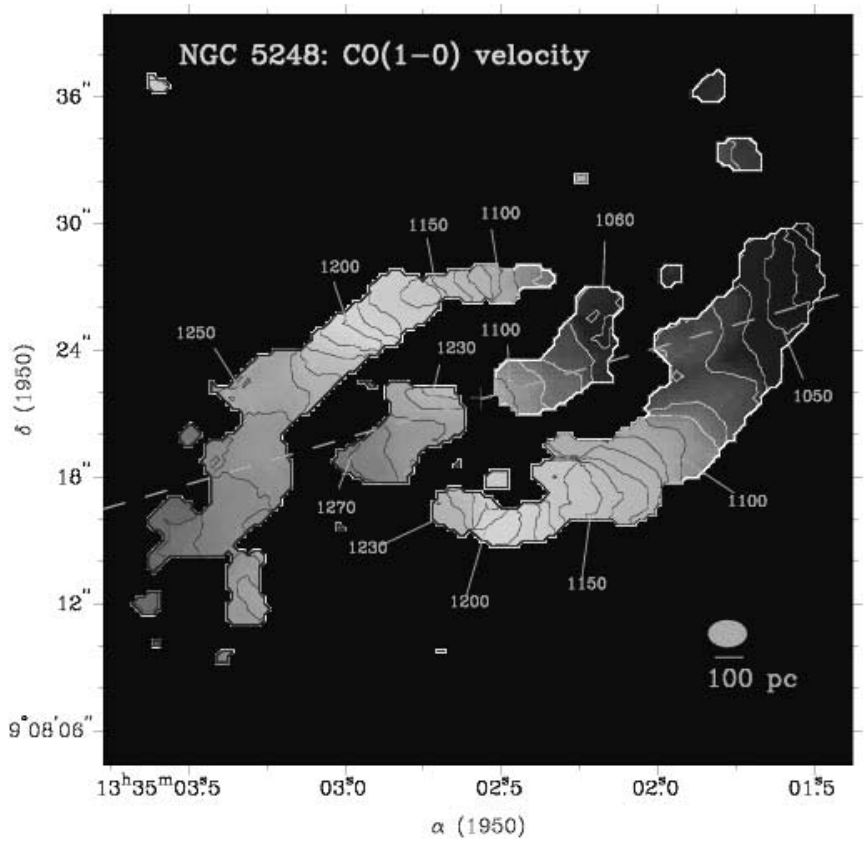

FIG. $11 a$

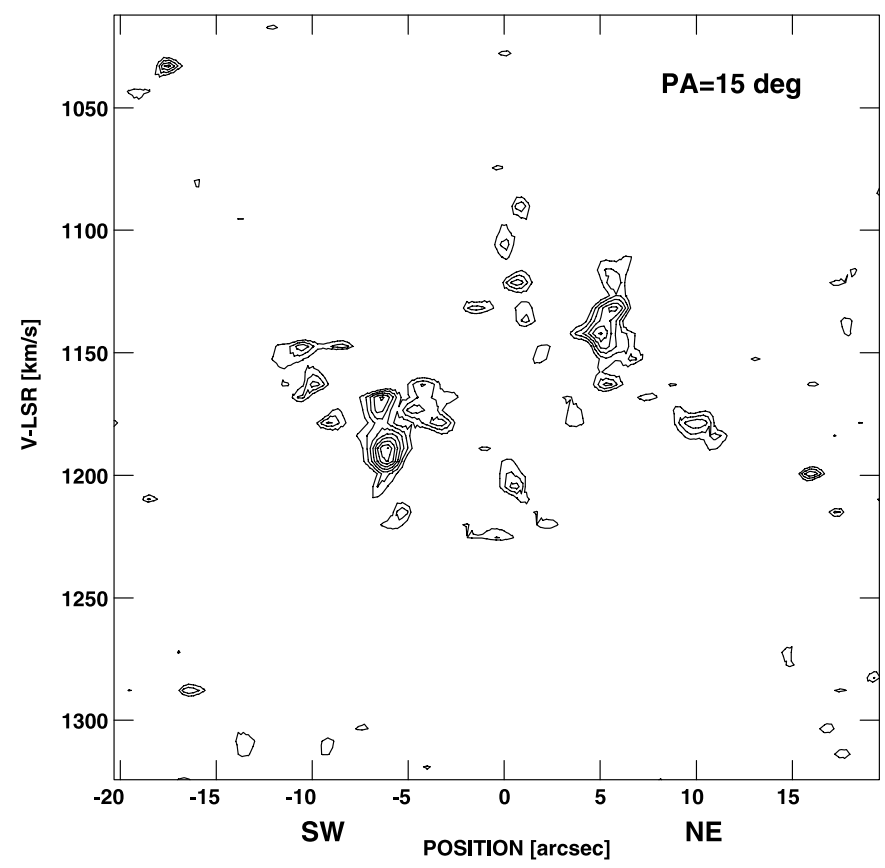

FIG. $11 b$

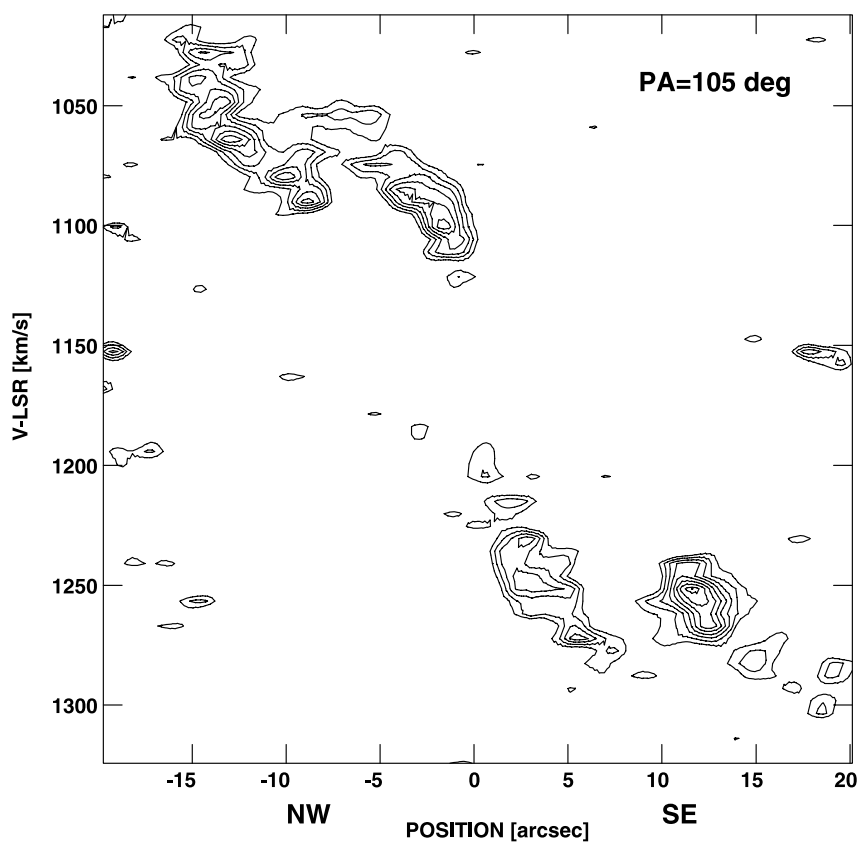

FIG. $11 c$

Fig. 11.- Molecular gas kinematics. (a) CO (1-0) intensity-weighted velocity field of the central 40". The 1".9 91 1"4 synthesized beam is marked. Contour levels range from 1000 to $1300 \mathrm{~km} \mathrm{~s}^{-1}$ and are plotted at intervals of $10 \mathrm{~km} \mathrm{~s}^{-1}$. (b) CO position-velocity cuts along the minor axis $\left(15^{\circ}\right)$. (c) CO position-velocity cut along the line of nodes $\left(105^{\circ}\right)$. [See the electronic edition of the Journal for a color version of Fig. 11 a.]

also been seen in observations of spiral arms in disks (e.g., Allen, Atherton, \& Tilanus 1986; Tilanus \& Allen 1989, 1991). If the gas in the central region is rotating clockwise like the stars, then the blueshifts and redshifts on the northwest and southeast side of the major axis, respectively (Fig. $11 a$ ), imply that the northeast side of the disk is the far side. This orientation is consistent with the dust lanes in the $J-K$ map (Fig. 4b) being redder on the western side than on the eastern side.

\section{EVOLUTION IN THE INNER KILOPARSEC OF NGC 5248}

We now take a closer look at evolution in the inner kiloparsec of NGC 5248 using the information synthesized from our high-resolution $\mathrm{CO}(1-0), J-K$, and $K_{s}$ observations, along with published HST images (Maoz et al. 2001). In particular, we focus here on the question of feeding the starburst ring with cold molecular fuel, the local SF properties, and the interplay of the SSCs with the interstellar medium. 


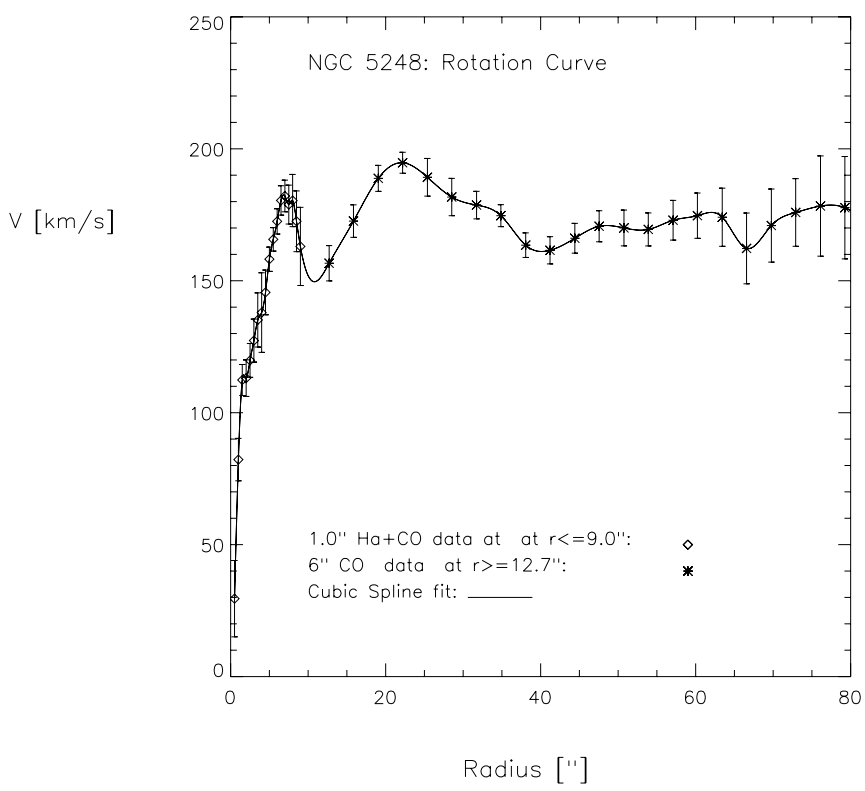

FIG. 12.- Rotation curve. Shown is the rotation curve from the center to $80^{\prime \prime}$, already corrected for an assumed inclination of $40^{\circ}$. It is based on the high-resolution Fabry-Perot $\mathrm{H} \alpha$ and OVRO CO (1-0) data out to $10^{\prime \prime}$ and BIMA data of lower resolution from $13^{\prime \prime}$ to $80^{\prime \prime}$. We interpolated between data points with a cubic spline fit. The rotation curve is likely contaminated by noncircular motions between $8^{\prime \prime}$ and $11^{\prime \prime}$ and between $12^{\prime \prime}$ and $18^{\prime \prime}$ (see text; $§ 5)$.

The integrated $\mathrm{H} \alpha+\left[\mathrm{N}_{\mathrm{II}}\right]$ luminosity in the inner kiloparsec radius of NGC 5248, after an extinction correction based on a dust screen model, is $\sim 2 \times 10^{41} \mathrm{ergs} \mathrm{s}^{-1}$ (Maoz et al. 2001). It corresponds to a current SFR of $2.1 M_{\odot} \mathrm{yr}^{-1}$, assuming an extended Miller-Scalo IMF, stellar masses from 0.1 to $100 M_{\odot}$, and a case B recombination. The true SFR could be higher if there is heavy internal extinction within the $\mathrm{H}$ II regions, but its upper limit is constrained by the global SFR of $3.2 M_{\odot} \mathrm{yr}^{-1}$, estimated for the entire galaxy. The latter rate is derived from the total far-infrared luminosity and low-resolution radio continuum data, following the prescription of Condon (1992). Table 4 summarizes SFR estimates based on different tracers. Our estimate of $2.1 M_{\odot} \mathrm{yr}^{-1}$ is also consistent with other circumnuclear properties. An average massive SFR of order $0.5 M_{\odot} \mathrm{yr}^{-1}$ or a total average SFR of $1.5 M_{\odot} \mathrm{yr}^{-1}$ is suggested by the young age (10-40 Myr; Maoz et al. 2001) and total mass (several times $10^{6} M_{\odot}$; Maoz et al. 2001) of the optically visible stellar clusters. The cluster ages are consistent with the age (8-10 Myr) of the $K$-band arms delineating the brightest SSCs (§ 4). A similar average circumnuclear SFR of $1.5 M_{\odot}$ $\mathrm{yr}^{-1}$ was derived by Elmegreen et al. (1997) based on the ages and masses of "hot spots" estimated from lower resolution NIR data.

The HST $\mathrm{H} \alpha$ (Fig. 6) image shows that most of the $\mathrm{H} \alpha$ emission originates from the circumnuclear ring of $\mathrm{H}$ II regions at a radius of $5^{\prime \prime}$ and the nuclear $\mathrm{H} \alpha$ ring at a radius of 1 ".25. Although the $\mathrm{CO}$ arms contain most $\left(1.2 \times 10^{9}\right.$ $M_{\odot}$ ) of the circumnuclear molecular gas, they show only patchy $\mathrm{H} \alpha$ (Fig. 5) and $\mathrm{Pa} \alpha$ (Maoz et al. 2001) emission. In fact, less than $10 \%$ of the $\mathrm{H} \alpha$ flux within the inner kiloparsec radius originates in the $\mathrm{CO}$ arms. In the western $\mathrm{CO}$ arm, $\mathrm{H} \alpha$ emission exists from $11^{\prime \prime}$ to $13^{\prime \prime}$ and from $16^{\prime \prime}$ to $19^{\prime \prime}$, in the same regions where very faint $K$-band emission is present $(\S 4)$. It is unlikely that the lack of optically visible
TABLE 4

SFR FROM DiFFERENT TRACERS

\begin{tabular}{|c|c|}
\hline Parameter & Value \\
\hline$L(1.4 \mathrm{GHz})^{\mathrm{a}}\left(\mathrm{W} \mathrm{Hz}^{-1}\right) .$. & $3.7 \times 10^{21}$ \\
\hline $\operatorname{SFR}(1.4 \mathrm{GHz})\left(M \mathrm{yr}^{-1}\right)$ & 3.2 (global) \\
\hline 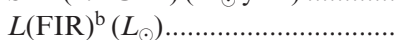 & $8.1 \times 10^{9}$ \\
\hline 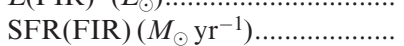 & 3.1 (global) \\
\hline$L(\mathrm{H} \alpha)^{\mathrm{c}}\left(\mathrm{ergs} \mathrm{s}^{-1}\right)$ & $2.3 \times 10^{41}$ \\
\hline $\operatorname{SFR}(\mathrm{H} \alpha)\left(M_{\odot} \mathrm{yr}^{-1}\right) \ldots \ldots \ldots \ldots \ldots$ & 2.1 (circumnuclear) \\
\hline $\operatorname{SFR}(K \text { band })^{\mathrm{d}}\left(M_{\odot} \mathrm{yr}^{-1}\right) \ldots \ldots$ & 1.5 (circumnuclear) \\
\hline
\end{tabular}

Note.-The table shows the derived SFRs using different tracers as described in the text. The term "global" refers to the entire galaxy, while the term "circumnuclear" refers to the inner $400 \mathrm{pc}$ radius, including the two rings of SF.

a Radio continuum luminosity density at $1.4 \mathrm{GHz}$ from Condon et al. 1990.

b FIR luminosity calculated from the IRAS 60 and 100 $\mu \mathrm{m}$ flux density following Helou et al. 1988.

${ }^{c}$ Extinction-corrected $\mathrm{H} \alpha$ luminosity from $H S T$ WFPC2 data (Maoz et al. 2001).

d Average SFR based on ages and masses of "hot spots" derived from NIR colors (Elmegreen et al. 1997).

$\mathrm{SF}$ along the $\mathrm{CO}$ arms is caused only by extinction, since the $\mathrm{Pa} \alpha$ and $K_{s}$ images do not reveal significantly more emission. High-resolution radio continuum observations, being relatively unaffected by dust, would have helped to address the question of obscured SF further. Unfortunately, the only existing high-resolution (1".5) observations do not have enough sensitivity to detect any emission in the central $40^{\prime \prime}$ (J. Wrobel \& J. Condon 2001, private communication). Thus, we have to consider the possibility that SF may be truly inhibited in the $\mathrm{CO}$ arms. One factor leading to this inhibition may be the shear induced by the noncircular kinematics in the $\mathrm{CO}$ arms ( $(5)$. The situation is reminiscent of the inner few kiloparsecs of M100 where two massive trailing $\mathrm{CO}$ arms with strong streaming motions (e.g., Sakamoto et al. 1995) host a large amount $\left(1.9 \times 10^{9} M_{\odot}\right)$ of molecular gas but have comparatively little star formation, below 1 $M_{\odot} \mathrm{yr}^{-1}$ (e.g., Knapen et al. 1995a). A low SFR per unit mass of molecular gas is also seen in the extended gas with noncircular kinematics along the bar in NGC 4569 (Jogee 1999) and NGC 7479 (Laine et al. 1999a). Another possibility is that the local gas surface density in the $\mathrm{CO}$ arms of NGC 5248, although high $\left(500-1500 M_{\odot} \mathrm{pc}^{-2}\right)$, is still below the critical density required to trigger widespread SF along the arms (see $\S 7.2$ for a dynamical argument related to this point).

A more detailed look at the properties of the $\mathrm{H}$ II regions and SSCs in relation to the molecular gas shows an interesting evolutionary effect. Table 5 lists several properties of emission-line complexes in NGC 5248, as derived by Maoz et al. (2001). For simplicity, we adopt the same identification numbers as used by these authors. The $\mathrm{H}$ II regions are listed in Table 5 in order of decreasing extinction, as judged by the $\mathrm{H} \alpha+\left[\mathrm{N}_{\text {II }}\right] / \mathrm{Pa} \alpha$ flux ratio. A value of $\sim 10$ corresponds to a case $\mathrm{B}$ recombination and no reddening, while values below 1 correspond to 3-4 mag of foreground dust extinction. Most $\mathrm{H}$ II regions in the starburst ring of NGC 5248 have intermediate values of $2-5$, corresponding to an extinction of 1-2 mag. The equivalent width of the Pa $\alpha$ line $\left[f(\operatorname{Pa} \alpha) / f_{\lambda}(1.6 \mu \mathrm{m})\right]$, listed in column (6), is a measure of the ratio of $\mathrm{O}$ stars to $\mathrm{K}$ and $\mathrm{M}$ giants and supergiants. It is of 
TABLE 5

EMISSION-LINE COMPLEXES IN NGC 5248

\begin{tabular}{|c|c|c|c|c|c|c|c|}
\hline $\begin{array}{l}\text { ID } \\
\text { (1) }\end{array}$ & $\begin{array}{c}\Delta \alpha \\
(\operatorname{arcsec}) \\
(2)\end{array}$ & $\begin{array}{c}\Delta \delta \\
(\operatorname{arcsec}) \\
(3)\end{array}$ & $\begin{array}{c}\text { Radius } \\
\text { (arcsec) } \\
(4)\end{array}$ & $\begin{array}{c}f\left(\mathrm{H} \alpha+\left[\mathrm{N} \mathrm{II}_{\text {II }}\right]\right) / f(\mathrm{~Pa} \alpha) \\
(5)\end{array}$ & $\begin{array}{c}f(\mathrm{~Pa} \alpha) / f_{\lambda}(1.6 \mu \mathrm{m}) \\
(\AA) \\
(6)\end{array}$ & $\begin{array}{c}f(\mathrm{H} \alpha) \\
\left(\times 10^{-16} \mathrm{ergs} \mathrm{s}^{-1} \mathrm{~cm}^{-2}\right) \\
(7)\end{array}$ & $\begin{array}{c}f(\mathrm{~Pa} \alpha) \\
\left(\times 10^{-16} \mathrm{ergs} \mathrm{s}^{-1} \mathrm{~cm}^{-2}\right) \\
(8)\end{array}$ \\
\hline $3 \ldots \ldots \ldots$ & 5.19 & 4.24 & 0.18 & 0.2 & 361 & 1.2 & 4.9 \\
\hline $4 \ldots \ldots \ldots$ & 5.73 & 4.03 & 0.18 & 0.3 & 515 & 1.9 & 6.8 \\
\hline $16 \ldots \ldots$ & -6.70 & -2.75 & 0.36 & 0.4 & 566 & 8.8 & 21.4 \\
\hline $9 \ldots \ldots \ldots$ & 10.18 & -0.5 & 0.18 & 1.1 & 943 & 7.8 & 6.8 \\
\hline $11 \ldots \ldots$. & 5.50 & -2.69 & 0.27 & 1.5 & 89 & 17.5 & 11.7 \\
\hline $13 \ldots \ldots$. & 0.98 & -6.24 & 0.32 & 1.6 & 170 & 21.8 & 13.6 \\
\hline $15 \ldots \ldots$. & -5.01 & -2.31 & 0.36 & 1.7 & 121 & 33.8 & 20.4 \\
\hline $2 \ldots \ldots \ldots$ & 2.97 & 2.99 & 0.18 & 1.9 & 149 & 16.9 & 8.8 \\
\hline $10 \ldots \ldots$ & 6.84 & -1.72 & 0.18 & 2.5 & 146 & 12.1 & 4.9 \\
\hline $5 \ldots \ldots \ldots$ & 6.04 & 3.21 & 0.18 & 2.5 & 349 & 17.0 & 6.8 \\
\hline $1 \ldots \ldots \ldots$ & 3.25 & 4.61 & 0.32 & 2.9 & 111 & 30.6 & 10.7 \\
\hline $18 \ldots \ldots$. & -3.74 & 2.74 & 0.23 & 3.2 & 58 & 21.8 & 6.8 \\
\hline $20 \ldots \ldots$ & -2.37 & 7.44 & 0.18 & 3.5 & 186 & 13.6 & 3.9 \\
\hline $7 \ldots \ldots \ldots$ & 4.52 & 0.62 & 0.32 & 3.7 & 41 & 35.8 & 9.7 \\
\hline $19 \ldots \ldots$. & -3.28 & 3.79 & 0.23 & 3.8 & 45 & 18.7 & 4.9 \\
\hline $17 \ldots \ldots$. & -6.21 & 0.93 & 0.36 & 3.9 & 58 & 45.2 & 11.7 \\
\hline $6 \ldots \ldots \ldots$ & 3.65 & 0.96 & 0.27 & 4.0 & 47 & 35.2 & 8.8 \\
\hline $12 \ldots \ldots \ldots$ & 4.10 & -3.90 & 0.45 & 4.1 & 87 & 104.7 & 25.3 \\
\hline $21 \ldots \ldots \ldots$ & -7.89 & 4.16 & 0.18 & 4.9 & 80 & 33.6 & 6.8 \\
\hline $14 \ldots \ldots \ldots$ & -2.32 & -3.93 & 0.91 & 5.0 & 61 & 358.0 & 72.0 \\
\hline $8 \ldots \ldots \ldots$ & 4.50 & -0.54 & 0.23 & 7.0 & 27 & 27.1 & 3.9 \\
\hline
\end{tabular}

Note.-The emission-line complexes are listed in order of decreasing extinction, as measured by the $\mathrm{H} \alpha+[\mathrm{N}$ II $] / \mathrm{Pa} \alpha$ flux ratio (Maoz et al. 2001). Col. (1): Identification number for the emission-line complex used by Maoz et al. 2001. Cols. (2) and (3): Offset along the R.A. and decl. direction from the galaxy nucleus whose pixel coordinates are (422".16, 396".02). Col. (4): Angular radius of the circular aperture used for flux measurements. Col. (5): The $\mathrm{H} \alpha+[\mathrm{N} \mathrm{II}] / \mathrm{Pa} \alpha$ flux ratio, which is a measure of extinction. Col. (6): The equivalent width of the Pa $\alpha$ line, defined as the flux ratio of the Pa $\alpha$ line and the continuum in the $1.6 \mu \mathrm{m}$ band. This is an age indicator for a single burst model. Cols. (7) and (8): The $\mathrm{H} \alpha+[\mathrm{N}$ II] and Pa $\alpha$ line fluxes without any correction for extinction. At the distance of $15.3 \mathrm{Mpc}$ for NGC $5248,10^{-16} \mathrm{ergs} \mathrm{s}^{-1} \mathrm{~cm}^{-2}$ corresponds to a luminosity of $2.8 \times 10^{36} \mathrm{ergs} \mathrm{s}^{-1}$.

order $400 \AA$ in the first few Myr and falls by 2 orders of magnitude within $10 \mathrm{Myr}$ (Leitherer et al. 1999; Maoz et al. 2001) under the assumption of a single burst model whose SFR decays exponentially on a timescale of $1 \mathrm{Myr}$. While the $\mathrm{Pa} \alpha$ equivalent width cannot be used as a detailed tracer of age, it is very likely that complexes with very large $\mathrm{Pa} \alpha$ equivalent widths of 349-943 $\AA$ (such as 3, 4, 16, and 9) are on average younger than complexes with equivalent widths below $50 \AA$ (e.g., 7, 19, 6, and 8).

The four emission-line complexes $(3,4,16$, and 9) with the largest extinction turn out to have large $\mathrm{Pa} \alpha$ equivalent widths (361-943 $\AA$ ) indicative of a young stellar population a few Myr old, but they do not show any optically visible continuum sources (Fig. 6). This suggests that the young stellar populations associated with these $\mathrm{H}$ II regions are obscured at optical wavelengths by gas and dust. In strong support of this suggestion, we find that these $\mathrm{H}$ in regions are located in the $\mathrm{CO}$ arms near bright $\mathrm{CO}$ peaks having local gas surface densities of $600-1500 M_{\odot} \mathrm{pc}^{-2}$. A similar avoidance between line-emitting regions and continuum sources has also been noted in the Antennae (Whitmore et al. 1999), NGC 2903 (Alonso-Herrero, Ryder, \& Knapen 2001), and several starbursts (Buta et al. 2000). NGC 5248 also hosts many $\mathrm{H}$ II regions $(8,14,21,12,6,17,19,7,18,20)$ with $\mathrm{H} \alpha+\left[\mathrm{N}_{\text {II }}\right] / \mathrm{Pa} \alpha$ flux ratios $\geq 3$ and small $\mathrm{Pa} \alpha$ equivalent widths (25-90 A). These $\mathrm{H}$ II regions (Fig. 6) lie near UV bright stellar clusters (Fig. 7), most of which have moderate extinction $\left(0 \mathrm{mag}<A_{V}<1 \mathrm{mag}\right)$, masses of several times $10^{3}-10^{5} M_{\odot}$, and ages typically in the range 10-40 Myr (Maoz et al. 2001). The ionized gas is often distributed in the form of shells and bubbles that surround the young stellar clusters, similar to what is observed in NGC 1569
(Hunter et al. 2000), NGC 5253 (Strickland \& Stevens 1999), and NGC 4214 (Maíz-Apellániz et al. 1999). This suggests that stellar winds and shocks from supernovae clear out the gas and dust. In support of this scenario, we find that in NGC 5248 most of these moderately extincted $\mathrm{H}$ II regions and optically visible SSCs are associated with low levels of $\mathrm{CO}$ emission (Figs. 6 and 7). Furthermore, we find that the molecular gas around the brightest complex of $\mathrm{H}$ II regions (14) has a bubble morphology suggestive of an evacuated cavity (Fig. 6). Taken together, the distributions of molecular gas, ionized gas, and stellar clusters in NGC 5248 evoke a scenario where young star-forming regions form within dense gas complexes, and subsequent stellar winds and supernovae efficiently clear out the gas on timescales of less than a few million years.

The origin of the $\mathrm{CO}$ and dust spirals will be discussed in $\S 7$ where we shall demonstrate that they lie well inside the outer ILR (OILR) of the bar. We discuss here the gas inflow rate from the $\mathrm{CO}$ arms into the star-forming ring at $5^{\prime \prime}$. The net inflow rate depends on the mass density profile and the vector sum of different velocity components at each radius. From a kinematic standpoint, the curvatures in the isovelocity contours, the local radial inflow streaming motions of $25-40 \mathrm{~km} \mathrm{~s}^{-1}$ along the kinematic minor axis, and the noncircular azimuthal streaming motions between $12^{\prime \prime}$ and $18^{\prime \prime}$ along the major axis $(\S 5)$ amply demonstrate that strong noncircular streaming motions exist in the $\mathrm{CO}$ arms. If one assumes an average mass density of $600 M_{\odot} \mathrm{pc}^{-2}$, a local radial velocity of $15 \mathrm{~km} \mathrm{~s}^{-1}$, and a width of $200 \mathrm{pc}$ for each $\mathrm{CO}$ arm, the implied gas mass inflow rate along the $\mathrm{CO}$ arms is a few $M_{\odot} \mathrm{yr}^{-1}$. This estimate provides an upper limit to the mass inflow rate from the $\mathrm{CO}$ arms into the star-forming 
ring because the gas flow is expected to diverge and form a "spray-type" flow (see $\S 7.2$ ) after the $\mathrm{CO}$ arms cross the bar minor axis and approach the star-forming ring. As a result of the spray-type flow, a significant fraction of the gas inflowing in one $\mathrm{CO}$ arm can overshoot the ring and enter the other arm, taking a longer time to reach the ring. The estimated upper limit of a few $M_{\odot} \mathrm{yr}^{-1}$ for the gas mass inflow rate along the $\mathrm{CO}$ arms is comparable to the current SFR of $1-2 M_{\odot} \mathrm{yr}^{-1}$ in the starburst ring ( $(6)$. Other estimates of the inflow rate can be made by calculating the gravitational torque exerted on the gas by the stars or by running tailored hydrodynamical simulations. These methods also carry large uncertainties as they depend on the exact shape of the underlying gravitational potential, as well as on uncertain parameters such as the viscosity, bar pattern speed, sound speed, simplified approximations of multiphase medium, and SF feedback.

\section{GASDYNAMICS IN THE CENTRAL KILOPARSEC OF NGC 5248}

\subsection{Gas Response and Dynamical Resonances}

A barred potential is made up of different families of periodic stellar orbits, characterized by a (conserved) Jacobi energy, $E_{\mathrm{J}}$, a combination of energy and angular momentum (e.g., Binney \& Tremaine 1987). The most important families are those aligned with the bar major axis (so-called $x_{1}$ orbits) or with its minor axis ( $x_{2}$ orbits; Contopoulos \& Papayannopoulos 1980). The $x_{1}$ family extends between the center and the bar's corotation radius. The $x_{2}$ family appears between the center and the ILR if a single ILR exists, or between the inner and outer ILRs if two ILRs exist. When $x_{2}$ orbits form, they are preferentially populated at each $E_{\mathrm{J}}$. Hence, the orientation of populated periodic orbits changes by $\pi / 2$ at each resonance.

The abrupt change in orientation at each resonance is restricted to (collisionless) stellar orbits. The gas-populated orbits can change their orientation only gradually as a result of shocks induced by the finite gas pressure. Therefore, the gas response to bar torquing leads to the formation of largescale offset shocks and a subsequent gas inflow, which slows down after crossing a resonance. This results in the formation of nuclear rings, each associated with a parent ILR. Generally, no one-to-one correspondence is expected between the existence of star-forming (i.e., blue) rings and ILRs, as rings can merge (Heller, Shlosman, \& Englmaier 2001) and/or age, becoming devoid of star formation (Shlosman 1999; Erwin, Beltran, \& Beckman 2001). Knapen et al. (1995a) and Shlosman (1996) have investigated locations of nuclear rings with respect to ILRs, concluding that the outer ILR is devoid of any pronounced SF because the associated ring sits well inside this resonance. The explanation of this phenomenon is closely tied to the rate of dissipation and the sound speed in the gas, as well as the bar strength, which defines the shape of the $x_{2}$ orbits and controls which outermost $x_{2}$ orbits get populated. At the same time, if an inner ILR (IILR) exists, the star-forming ring is positioned exactly at this resonance, as a result of the reversal of gravitational torques.

The exact locations and even the number of ILRs can be inferred reliably only from nonlinear orbit analysis (e.g., Heller \& Shlosman 1996) based on the knowledge of the galactic potential. In this paper we use approximate meth- ods that rely on SF morphology and isophote fitting. The first method relies on the properties of nuclear rings discussed above. The dominant circumnuclear ring at a radius of $5^{\prime \prime}(375 \mathrm{pc})$ shows that NGC 5248 has at least one ILR. Whether this galaxy has a second ILR near $r=1$ ". 25 , where the second inner $\mathrm{H} \alpha$ ring resides, is not entirely clear. In principle, the latter ring may not be tied to any dynamical resonance but could represent a superbubble driven by a recent starburst event in the nucleus. However, there is no evidence of a bright young central cluster in NGC 5248 (D. Maoz 2001, private communication), and the bright background light from the bulge makes it hard to identify a faint central source. Hence, it is plausible that NGC 5248 hosts both an OILR and an IILR.

A complementary isophote fitting to the deprojected $R$-band and $K_{s}$ (Fig. 10b) images also provides a guide to the location of the OILR. The isophotes change gradually from being oriented along the bar major axis around $\sim 115^{\prime \prime}$ to being perpendicular to the bar around $35^{\prime \prime}$. This gradual twist rather than an abrupt change in orientation results from the unusually prominent young spiral arms inside the bar corotation. The isophote fits provide some insight into locations of dominant $x_{1}$ and $x_{2}$ families of orbits, oriented parallel and perpendicular to the bar, respectively. Those corresponding to the outermost $x_{2}$ orbits cross the bar minor axis at $\sim 35^{\prime \prime}$ and the bar major axis at $\sim 26^{\prime \prime}$. These locations can be taken as approximate positions of the OILR along the major and minor axes of the bar. The point $\mathrm{P} 2^{\prime}$ in Figure $13 a$ and point $\mathrm{P} 2$ in Figure $13 b$ denote the approximate position of the OILR along the bar minor axis. The position of the OILR is further confirmed by the azimuthal twist of the stellar spiral arms by $\pi / 2$ between the OILR and the CR and an additional $\pi / 2$ between the $\mathrm{CR}$ and the OLR (Fig. 1) The latter is estimated to lie around $\sim 230^{\prime \prime}$, at the edge of the outer disk where the spiral arms end $(\S 3)$. Furthermore, Laine et al. (2002) have analyzed a subsample of barred galaxies among 112 disks, concluding that the ratio of $r_{\text {ILR }} / D_{25}$ is about 0.06 , where $r_{\text {ILR }}$ is taken along the bar major axis. For comparison, this ratio is 0.07 in NGC 5248 , assuming $r_{\text {ILR }}=26^{\prime \prime}$ and $D_{25}=370^{\prime \prime}$ (Table 1).

To summarize, we discuss briefly three possibilities concerning the number of ILRs in NGC 5248:

1. One ILR.-In this case, with the ILR at around $26^{\prime \prime}$ (2 $\mathrm{kpc}$ ), it is difficult to explain why the star-forming ring at 375 pc lies so deep inside the resonance. NGC 5248, therefore, would appear as an extreme case. Another limitation of this scenario is that it does not provide an explanation for the inner $\mathrm{H} \alpha$ ring at $95 \mathrm{pc}$.

2. Two ILRS.-In this case, the OILR is at $2 \mathrm{kpc}$ and the IILR at $95 \mathrm{pc}$. This option is attractive because, as required by numerical simulations, the OILR itself is devoid of pronounced SF and there is an inner star-forming ring at the IILR and an outer ring at $375 \mathrm{pc}$ between the ILRs. However, one discrepancy is that, in simulations, the IILR ring generally has more prominent SF than the outer ring, while the converse would appear to hold here.

3. Three ILRs.-In this case, the OILR is at $2 \mathrm{kpc}$, the IILR is at $375 \mathrm{pc}$, and the innermost resonance, hereafter $\mathrm{OILR}_{2}$, is at $\sim 200 \mathrm{pc}$, outside the ring at $95 \mathrm{pc}$. This option sets the more prominent ring with active SF, namely, the $375 \mathrm{pc}$ ring, at the IILR. A combination of high-resolution velocity fields and future detailed modeling can help distinguish between the different possibilities. Meanwhile, for 


\section{MODEL}
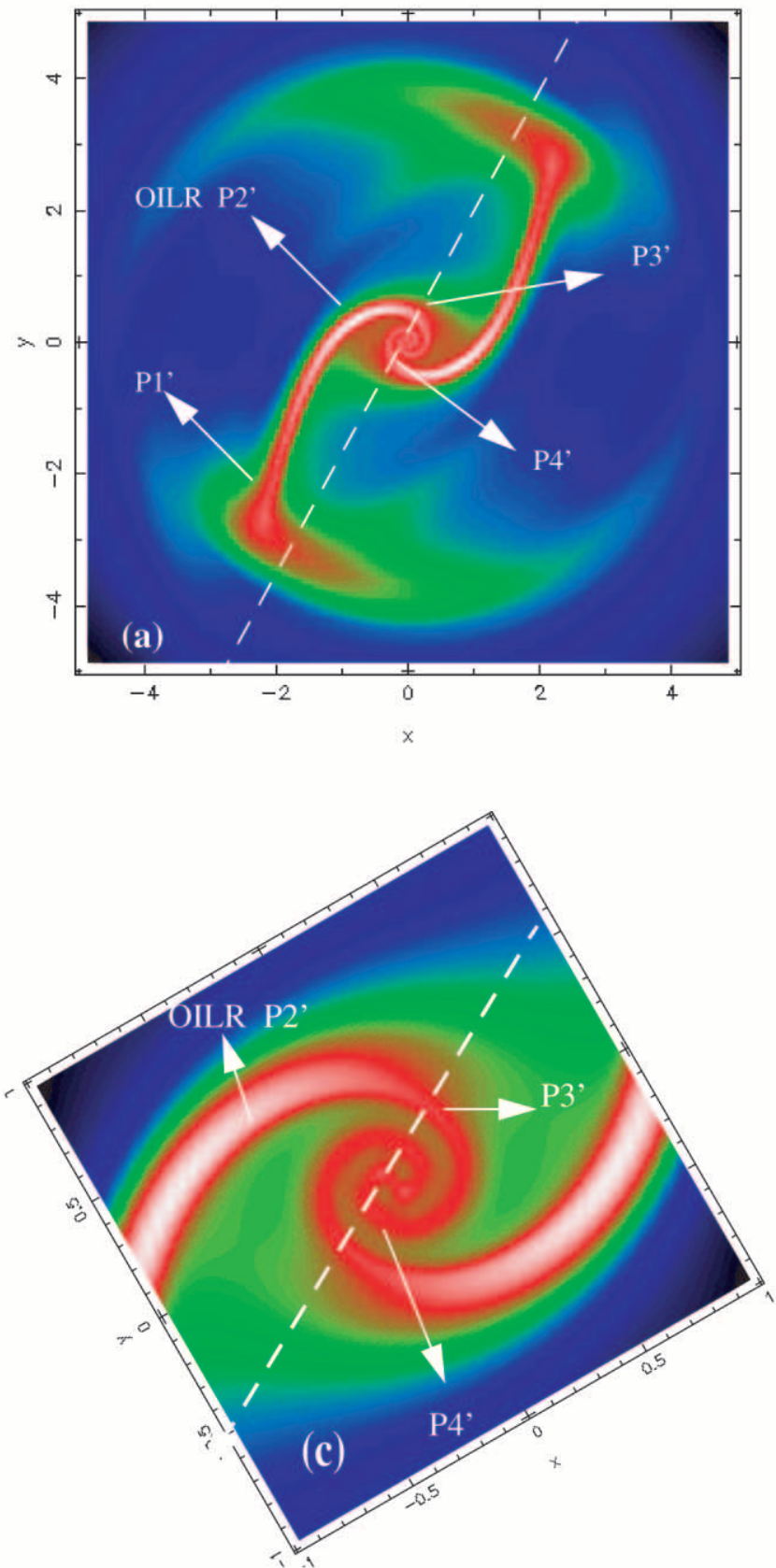

DATA
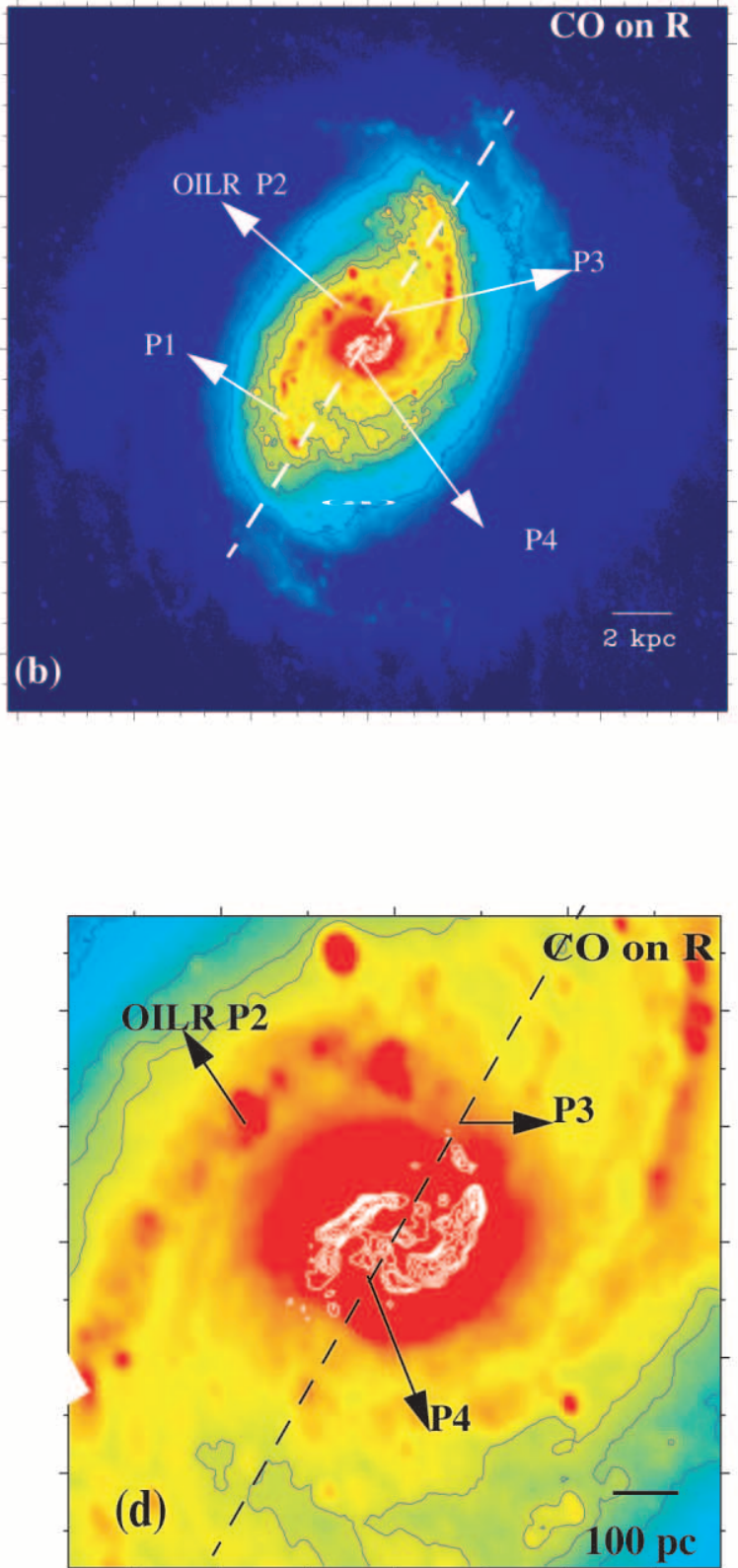

Fig. 13. - Comparison of the CO and $R$-band spirals with hydrodynamical models of bar-driven gaseous spiral density waves in NGC 5248. The steady state gas response in models of bar-driven gaseous SDW is shown $(a)$ from the central regions out to the end of the bar and $(c)$ only in the inner few kiloparsecs, deep inside the OILR of the bar. Points $\mathrm{P}^{\prime}{ }^{\prime}-\mathrm{P} 4{ }^{\prime}$ in the models correspond to points P1-P4 in the data. The orientation of the large-scale stellar bar is shown by a dotted line. Strong bar shocks around the OILR excite a trailing nonlinear high-amplitude gaseous SDW that weakens rapidly as it travels inside the OILR. Under the right conditions, it can excite a linear low-amplitude gaseous SDW inside a transition radius $R_{t}$. This linear SDW can wind through an arbitrary angle because its shape is not dictated by the dominant stellar orbits. The deprojected CO map (white contours) of the central $40^{\prime \prime}(3.0 \mathrm{kpc})$ is overlaid on a large-scale $R$-band image. Regions of size $(b) 8^{\prime}(36 \mathrm{kpc})$ and $(d) 2^{\prime}(9 \mathrm{kpc})$ are shown. The two massive trailing CO spiral arms lie deep inside the OILR, cover more than $180^{\circ}$ in azimuth, and appear to correspond to the high-amplitude nonlinear gaseous SDW. The double-peaked CO feature F1 and the nuclear dust spiral that lie inside $4^{\prime \prime}$ appear to be associated with the linear gaseous SDW inside the transition radius $R_{t}$.

brevity, we shall work within the framework of two to three ILRs, as these models avoid most of the controversies.

Aside from the morphology of $\mathrm{H} \alpha$ rings, kinematic properties can in principle constrain the number and location of ILRs. For a weak bar where the linear epicyclic approximation is (very roughly) tolerable, we can estimate the location of ILRs if we have a reliable measure of the angular speed $\Omega$ and the epicyclic frequency $\kappa$. The latter quantity depends sensitively on the circular velocity as a function of radius. The rotation curve (Fig. 12) for NGC 5248 suffers from noncircular motions between $8^{\prime \prime}$ and $11^{\prime \prime}$ and between $12^{\prime \prime}$ and $18^{\prime \prime}(\S 5)$. In consequence, the linear resonance diagram is too noisy to provide meaningful estimates of the location of 
the ILRs. However, an upper limit to the bar pattern speed $\Omega_{p}$ can be inferred from it. $\Omega$ is of order $30 \mathrm{~km} \mathrm{~s}^{-1} \mathrm{kpc}^{-1}$ around a radius of $70^{\prime \prime}$, and for a flat rotation curve it must be even lower at the CR of $115^{\prime \prime}$ (Paper I and $\S 3$ ). The condition $\Omega=\Omega_{p}$ at CR, therefore, implies that the bar pattern speed is below $30 \mathrm{~km} \mathrm{~s}^{-1} \mathrm{kpc}^{-1}$. This low pattern speed will be used in $\S 7.2$.

An interesting, albeit speculative, possibility is that we may be observing a time-dependent rather than a quasistatic phenomenon in the disk of NGC 5248. Numerical modeling of the evolution, geometry, and associated SF of circumnuclear rings shows that when gas inflow rates along the bar are large, the ring is typically oval in shape and leads the bar by $\sim 50^{\circ}-60^{\circ}$ (Knapen et al. 1995a; Shlosman 1996). Once gas inflow rates slow down substantially, the circumnuclear ring acquires a round shape and a relatively uniform distribution of SF, typically after a characteristic timescale of order $10^{8} \mathrm{yr}$. In the deprojected $\mathrm{H} \alpha$ image of NGC 5248, the star-forming ring at $375 \mathrm{pc}$ has such a quasi-circular appearance, with star formation uniformly distributed azimuthally. Furthermore, a significant fraction of the stellar clusters in NGC 5248 have ages of order $100 \mathrm{Myr}$ (Maoz et al. 2001), consistent with the above characteristic evolutionary timescale. On the other hand, the massive $\mathrm{CO}$ arms with large noncircular motions joining the ring, the young age (10-20 Myr; Maoz et al. 2001) of many SSCs in the ring, and the young age (10 Myr; $\S 4)$ of the $K$-band arms that delineate them all point to a recent triggering of SF in the last 10-20 Myr. Taken together, the complicated morphology of the stars and ISM in the vicinity of ILRs and the range of ages seen in the stellar clusters allow for a strongly time-dependent evolution of the inner kiloparsec.

\subsection{Bar-driven Gas Density Waves inside the ILR}

The present work provides ample observational evidence in favor of continuity of grand-design spiral structure from the nuclear (subkiloparsec) to $10 \mathrm{kpc}$ scales in the barred galaxy NGC 5248. In particular, our observations ( $§ 3$ ) show that NGC 5248 hosts spiral structure in stars, dust, and gas over a striking 2 orders of magnitude in spatial scales. They also demonstrate that spiral structure in NGC 5248 winds up over a large azimuthal angle, at least $\pi$, deep inside the OILR $(\S 7.1)$. The two massive $\mathrm{CO}$ and dust spirals (Fig. $4 b$ ) extend between $20^{\prime \prime}$ and $5^{\prime \prime}$, connecting to the inner young $K$-band arms $(\S 3$; Fig. 8$)$ that continue from $8^{\prime \prime}$ to $3^{\prime \prime}$, delineating the SSCs (Fig. 7) in the $5^{\prime \prime}$ star-forming ring. Still closer to the center, between $1^{\prime \prime}$ and $4^{\prime \prime}$, reside the nuclear dust spiral, the double-peaked molecular feature, and the nuclear $\mathrm{H} \alpha$ ring ( $\S 3$ ). To account for the observed properties, we next compare NGC 5248 to theoretical models of gas response deep inside the ILR of a barred potential.

According to the density wave theory (e.g., Lin \& Shu 1964; Lin, Yuan, \& Shu 1969; Bertin et al. 1989a, 1989b), stellar density waves cannot propagate inward across the ILR and are damped at smaller radii. However, gaseous density waves, in principle, can exist at most radii, depending on the degree of self-gravity in the gas. If the gas selfgravity is ignored, the gaseous wave propagation is limited to the region between the ILRs (for the case of two ILRs) or to the region between the center and the ILR (for the case of one ILR). The propagation of gaseous density waves in the central regions of disk galaxies has attracted recent attention and has been investigated with hydrodynamical simula- tions (Englmaier \& Shlosman 2000). In these simulations, strong bar shocks that exist in the vicinity of the ILR(s) trigger gas density waves that propagate radially inward and are sheared by galactic rotation. The transition between the gaseous wave driven by the outer shocks and the lower amplitude wave that is propagating inward happens near the transition radius $R_{t}$, where shocks cross the bar major axis (see the point $\mathrm{P}^{\prime}$ in Fig. 13c). The simple explanation for the sudden drop in wave amplitude seems to be the existence of a spray-type flow in this region. In other words, the flow diverges here suddenly because of trajectory curvature, and the surface density drops as a result. This type of flow happens only when the wave approaches the major axis of the bar, while at other azimuths the flow streamlines converge. When gas self-gravity is neglected, $R_{t}$ should lie at the first crossing, at P3', as noted by Englmaier \& Shlosman (2000). Gas self-gravity will of course prevent the flow streamlines from diverging at $\mathrm{P} 3^{\prime}$.

Englmaier \& Shlosman (2000) ignored the contribution of self-gravity in their dispersion relationship for gaseous waves deep inside the ILRs of barred galaxies and in their modeling. This approach was motivated by the observed low arm/interarm contrast of $\sim 2$ and the lack of star formation along the nuclear dust spiral in NGC 5248, observed in $J-K$ between $1^{\prime \prime}$ and $4^{\prime \prime}$ (Laine et al. 1999b). The shape of the resulting gaseous spiral depends on two parameters: the degree of central concentration and the sound speed in the gas. One can obtain a range of shapes, from a tightly wound to an open one. The tightly wound spirals in fact degenerate into a featureless disk. The theoretical and numerically modeled shapes of the spirals and their amplitudes are found to agree well within the transition radius $R_{t}$. Outside this radius the theoretical curve cannot be used because it neglects the effects of gas self-gravity and noncircular orbits. The former leads to a singularity in the analytical expression for the pitch angle, $\tan i_{p} \sim 1 / R|k|$, at the ILR, where the wavevector $k \rightarrow 0$. This unphysical behavior can be corrected by adding the gravitational term $-2 \pi G \Sigma|k|$, where $\Sigma$ is the gas surface density, to the dispersion relationship (eq. [1] in Englmaier \& Shlosman). The prime motivation for introducing this correction in this paper is the existence of massive molecular (CO) arms with a large (arm/interarm) contrast in NGC 5248.

Figures $13 a$ and $13 c$ show the model gas response along the entire bar and inside the OILR, respectively. The corresponding observed spirals are shown, respectively, in Figures $13 b$ and $13 d$. The massive CO arms in NGC 5248 appear to correspond to the high-amplitude nonlinear gaseous waves propagating inward across the OILR, in which case they would have to lie outside the transition radius $R_{t}$. On the other hand, the double-peaked CO feature F1 and nuclear dust spiral, which lie interior to the starburst ring of $5^{\prime \prime}$ radius, appear to be associated with the linear wave. This suggests that $R_{t} \sim 5^{\prime \prime}-6^{\prime \prime}$. Note that the minimum of the spiral pitch angle $i_{p}$ (Fig. 14, top panel) lies near this estimated $R_{t}$.

The top panel of Figure 14 displays the observed (dotted line) pitch angles of gaseous spirals. The pitch angle of the dusty spiral is marked with a dot at $5^{\prime \prime}$. The solid line is a smooth eyeball fit to the observed pitch angle, which we used below for the self-gravitating case. In the case in which the gaseous density wave and large-scale bar have the same pattern speed, and when the self-gravity of the gas can be neglected, the model of Englmaier \& Shlosman (2000) pre- 


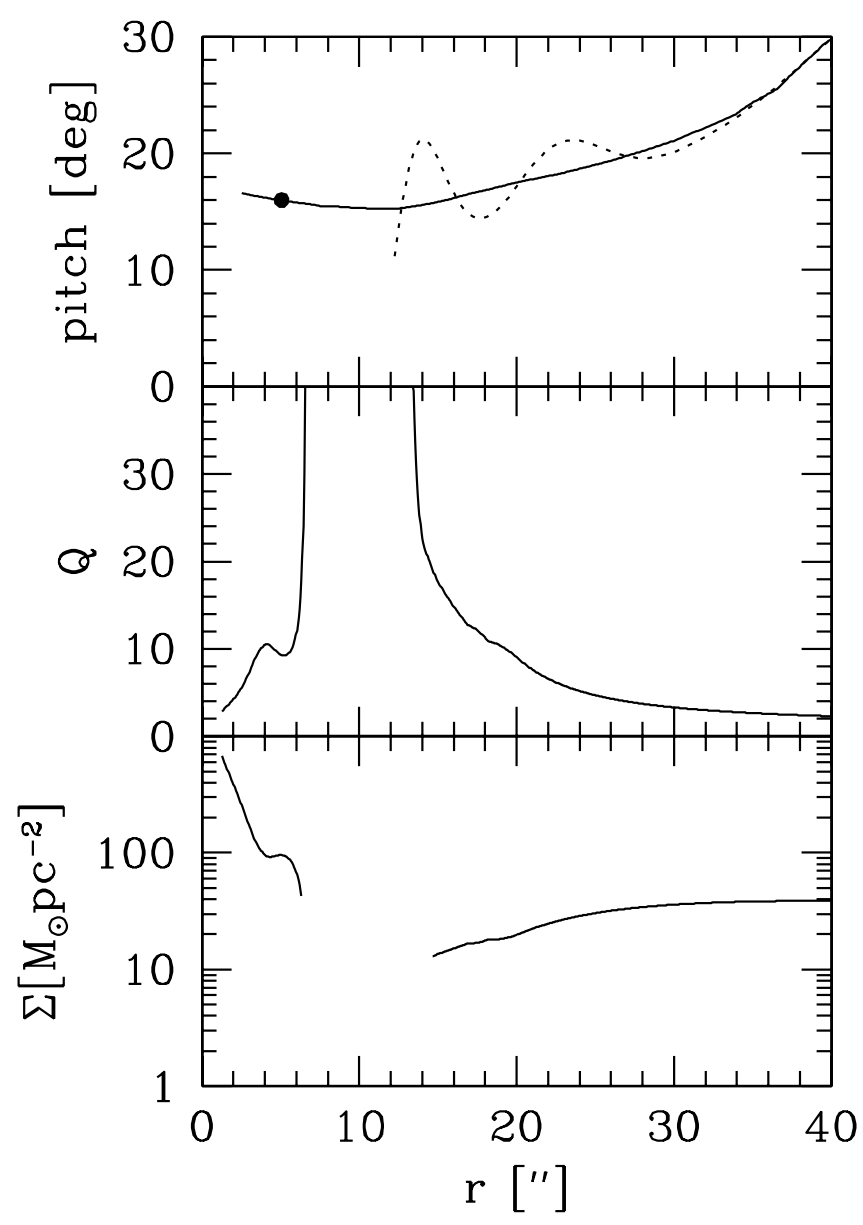

FIG. 14.-Comparison of observed and model pitch angles. Top: Observed pitch angle for the nuclear dusty spiral ( filled circle) and the $\mathrm{CO}$ spirals (dotted line), as well as the best eyeball fit to these data (solid line). For the eyeball fit, we computed the model including the self-gravity term shown in the other two panels based on the rotation curve of NGC 5248, a sound speed $v_{s}=16 \mathrm{~km} \mathrm{~s}^{-1}$, and a pattern speed $\Omega_{s}$ of $25 \mathrm{~km} \mathrm{~s}^{-1} \mathrm{kpc}^{-1}$. Middle: Effective Toomre parameter $Q$ (see the text) along the spiral arms. Bottom: Inferred gas surface density profile along the spiral arms. A transition radius, $R_{t}$, separates the outer nonlinear gas response from the linear response at smaller radii.

dicts a unique run of pitch angle with radius, once the sound speed, the pattern speed, and the rotation curve are specified. As the nonlinear gaseous spiral wave travels inside the OILR, the model predicts that its pitch angle will drop until it gets into resonance with the linear gaseous wave at $R_{t}$. Interior to $R_{t}$, the pitch angle is expected to increase. On the basis of this predicted behavior, the top panel of Figure 14 suggests that $R_{t}$ lies around $6^{\prime \prime}-12^{\prime \prime}$. One can discuss separately the behavior of two branches, for the radii smaller or larger than $R_{t}$. The left branch is formed by the dust nuclear spiral, while the right one is associated with the dusty spiral that follows the $\mathrm{CO}$ arms. Note that the pitch angle of the two trailing $\mathrm{CO}$ arms can be reliably measured only from a radius of $18^{\prime \prime}(1.3 \mathrm{kpc})$ to $6^{\prime \prime}(450 \mathrm{pc})$. From $6^{\prime \prime}$ and $3^{\prime \prime}$, the $\mathrm{CO}$ pitch angle cannot be reliably measured as the emission is too weak.

This region between $3^{\prime \prime}$ and $6^{\prime \prime}$ is where the spiral structure appears to continue in the form of $K$-band arms that delineate the SSCs in the starburst ring. As discussed in $\S 6$, the weak $\mathrm{CO}$ emission in this region is likely due to $\mathrm{CO}$ near the SSCs being cleared out by winds and supernovae, or dissoci- ated. Fortunately, the pitch angle of the nuclear dust spiral (Laine et al. 2001) provides empirical data between $1^{\prime \prime}$ and $4^{\prime \prime}$.

One can also infer the physical characteristics of gaseous spirals that result from the smooth eyeball fit to the observed pitch angle (solid line; Fig. 14, top panel) and the self-gravity term. These inferred characteristics include the Toomre $Q\left(\equiv v_{s} \kappa / \pi G \Sigma\right)$ parameter and the gas surface density. Here $v_{s}$ is the gas sound speed and $\kappa$ is the epicyclic frequency. The middle panel of Figure 14 displays the effective $Q$ for $v_{s}=15 \mathrm{~km} \mathrm{~s}^{-1}$ and the bar pattern speed $\Omega_{p}=25 \mathrm{~km}$ $\mathrm{s}^{-1} \mathrm{kpc}^{-1}$. This bar pattern speed is justified in $\S 7.1$. The $Q-$ curve tends asymptotically to infinity on both sides of the "gap" between 6".5 and 12". Larger values of $Q$ correspond to progressively less " self-gravity." This gap has a counterpart in the surface gas density profile (Fig. 14, bottom panel), where $\Sigma \rightarrow 0$. The gap in the above analysis displays the effect of the $5^{\prime \prime}$ ring and the associated $K$-band arms extending to about $8^{\prime \prime}$. One should not forget also that the noncircular motions are large in this region adjacent to the ring $(\S 5)$. Clearly, neglecting the contribution of the stellar component is not permitted here.

Our estimate for the position of $R_{t}$ in the top panel of Figure 14 can be independently tested. Namely, close to the OILR, large deviations from purely circular motions are expected (Fig. 15a). On the other hand, inside $R_{t}$, the model predicts an azimuthal flow speed that is close to the circular speed. Thus, around $R_{t}$, the observed and circular speed are expected to converge (Fig. 15a). Figures $15 b$ and $15 c$ show the observed difference between the circular speed curve and the $\mathrm{CO}$ velocity. The largest deviation from circular motions occurs between a radius of $7^{\prime \prime}$ and $10^{\prime \prime}$, and a sharp change is seen at about $6^{\prime \prime}$, where the observed and circular speeds converge. This supports our estimate of $R_{t} \sim 6^{\prime \prime}$. The kinematical properties are therefore consistent with the model predictions.

The above combination of multiwavelength observations and modeling suggests that when the right conditions are met, a bar can drive a gaseous spiral density wave (SDW) that winds over a large azimuthal angle and extends deep inside the ILR. The perturbations thus generated inside the OILR can be strong and self-gravitating, such as the massive $\mathrm{CO}$ arms in NGC 5248. Overall, the spiral structure induced by the bar may lead to an appreciable gas inflow rate relevant for the fueling of starburst activity in nuclear rings-a possibility strongly hinted at in NGC 5248 by the large noncircular motions in the $\mathrm{CO}$ arms and the massive recent $\mathrm{SF}$ along the young $K$-band spirals ( $\S 6$ ). A relatively low central mass concentration (evident through a shallow rotation curve), which prevents the spirals from winding up, is a crucial condition favorable to the propagation of the gaseous SDW deep inside the ILR. This condition is more easily met in late-type than in early-type spirals. Conversely, in the presence of a large central mass concentration, the models of Englmaier \& Shlosman (2000) show that the gaseous wave driven by the outer bar shocks can die away rapidly inside the ILR. This very condition, interestingly enough, leads to the formation of ILRs and favors the formation and dynamical decoupling of secondary nuclear bars by causing the inner regions of a bar to rotate much faster than primary bar pattern speed. We note that the rotation curve of NGC 5248 is shallower than that of many well-studied barred galaxies such as NGC 6951, NGC 4102 (Jogee 1999), NGC 2782 (Jogee et al. 1999), and M100 (Knapen et al. 

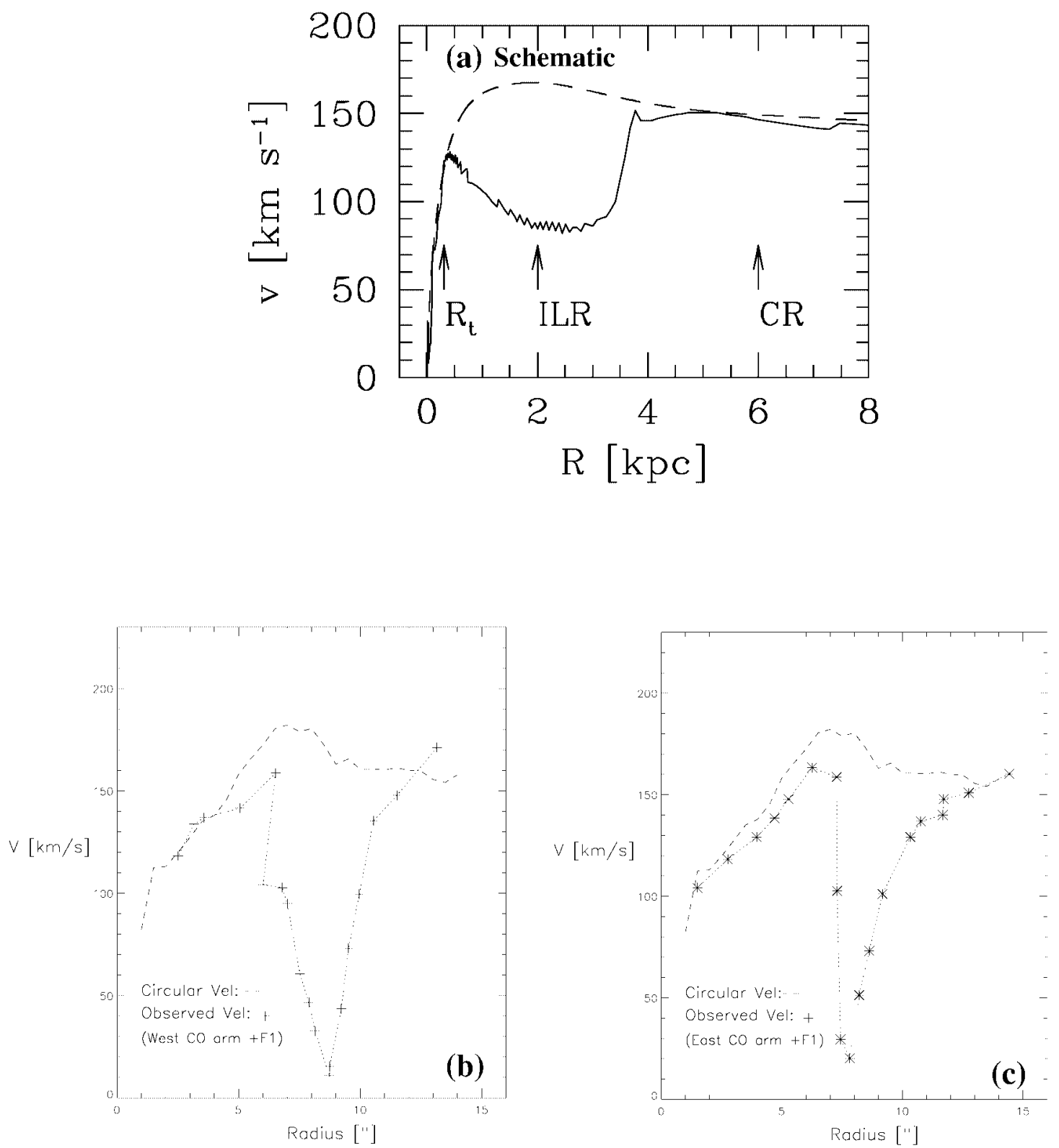

FIG. 15.-Comparison of observed and model kinematics. (a) Gas kinematics predicted by models of the gaseous SDW. Close to the OILR, where shocks from the bar perturb most, large deviations from purely circular motions are expected. Inside the transition radius $R_{t}$ the azimuthal speed is very close to the circular speed because the $x_{2}$ orbits are almost circular. A sharp change in behavior is expected at $R_{t}$. (b) Observed difference between the circular speed curve and the $\mathrm{CO}$ velocity. The velocity is measured along the western trailing $\mathrm{CO}$ spiral arm and the southern part of the double-peaked CO feature $\mathrm{F} 1$. The largest deviations from circular motions occur between $7^{\prime \prime}$ and $10^{\prime \prime}$, and a sharp change is seen at a radius of about $6^{\prime \prime}$ where the observed and circular speeds converge. (c) Same as in (b), but for the eastern trailing $\mathrm{CO}$ spiral arm and the northern part of the $\mathrm{CO}$ feature F1.

1995b). In NGC 5248, the offset spiral dust lanes and CO arms cover at least $180^{\circ}$ in azimuth inside the OILR before they join a starburst ring (Figs. 6 and 13d), while in NGC 6951 (Jogee 1999) and M100 (Knapen et al. 1995b) the corresponding azimuthal angle is only $\sim 90^{\circ}$. In NGC 2782 , which hosts a nuclear bar, the $\mathrm{CO}$ rotation curve has a turnover velocity of above $300 \mathrm{~km} \mathrm{~s}^{-1}$ and is much steeper than in NGC 5248 (Jogee et al. 1999).

\section{SUMMARY AND DISCUSSION}

We present a study of the grand-design spiral structure, gasdynamics, and circumnuclear SF in the nearby barred galaxy NGC 5248, based on a multiwavelength data set and theoretical modeling of gasdynamics. The extensive data set includes high-resolution $\left(1.9 \times 1{ }^{\prime \prime} 4\right)$ OVRO CO (1-0) observations, optical and NIR ground-based data, along with HST images and Fabry-Perot $\mathrm{H} \alpha$ observations. This study provides the best evidence to date for a strong dynamical coupling between the nuclear (subkiloparsec) region and the large-scale features in the outer galactic disk. It shows how bar-driven spiral structure in NGC 5248 extends over 2 orders of magnitude in spatial scales, shapes disk evolution, and fuels SF on progressively smaller scales.

The grand-design spiral structure is particularly evident in the dust spirals that cover at least $360^{\circ}$ in azimuth and continue from a radius of $70 \mathrm{kpc}$ down to $375 \mathrm{pc}$, penetrat- 
ing the OILR of the bar located at $2.0 \mathrm{kpc}$. Interior to the OILR, two massive trailing molecular spiral arms cover nearly $180^{\circ}$ in azimuth from a radius of $1.5 \mathrm{kpc}$ to $375 \mathrm{pc}$, where they feed a circumnuclear starburst ring. The molecular spirals are massive, containing $1.2 \times 10^{9} \mathrm{M}_{\odot}$ of gas, and show noncircular streaming motions of $20-40 \mathrm{~km} \mathrm{~s}^{-1}$. At a radius of $\sim 600 \mathrm{pc}$, they connect to two $K$-band arms that cross the starburst ring and continue down to $225 \mathrm{pc}$. The UV bright SSCs seen in the HST image lie along these $K$-band spirals. The particularly narrow width $(\sim 110 \mathrm{pc})$ of the $K$-band arms suggests a young dynamical age of $\sim 10$ Myr, comparable to the age of the SSCs (10-40 Myr; Maoz et al 2001) and that of the supergiants (8-10 Myr), which often dominate $K$-band emission in starburst regions. Altogether, the data suggest that the $K$-band arms are young, and recent SF in the ring at $375 \mathrm{pc}$ has been triggered by a bar-driven density wave. The density wave may have even propagated into the central $100 \mathrm{pc}$ since the $K$-band arms appear to connect to a grand-design nuclear dust spiral that continues inward from $\sim 225$ to $\sim 75$ pc (Laine et al. 1999b). This dust spiral crosses a second $\mathrm{H} \alpha$ ring of radius $95 \mathrm{pc}$ and a double-peaked molecular feature.

We estimate an SFR of about $2 M_{\odot} \mathrm{yr}^{-1}$ in the inner few kiloparsecs of NGC 5248. Only a small fraction of this SFR originates in the massive $\mathrm{CO}$ spiral arms although they contain most of the molecular gas. The shear induced by the noncircular kinematics, as well as subcritical gas densities, may be responsible for the low SF efficiency in the arms. The latter point is supported by modeling. We also find that the four emission-line complexes that have the largest extinction and line ratios consistent with an embedded, optically invisible, young stellar population, a few Myr old, are located in the massive $\mathrm{CO}$ arms, near bright $\mathrm{CO}$ peaks with large gas surface densities of $600-1500 M_{\odot} \mathrm{pc}^{-2}$. Conversely, visible SSCs that have moderate extinction $\left(0<A_{V}<1 \mathrm{mag}\right)$ lie in the starburst ring where they are associated with low levels of $\mathrm{CO}$ emission and are surrounded by shells of ionized gas. Overall, the distribution of molecular gas, ionized gas, and stellar clusters in NGC 5248 is consistent with a picture where young star-forming regions are born within dense gas complexes and subsequent stellar winds and supernovae efficiently clear out gas on timescales of less than a few million years.
There is mounting evidence from recent high-resolution (10-100 pc) ground-based and HST observations that grand-design dust and gas spirals are common in the inner kiloparsec of galaxies. In NGC 5248, a bar-driven SDW has led to massive CO spiral arms that cover a large range in radius inside the OILR and feed a starburst ring. To account for such massive perturbations, we have generalized the Englmaier \& Shlosman (2000) models of gaseous SDW by incorporating the effect of gas self-gravity. We thereafter find good agreement between the modeled and observed gas morphology, gas kinematics, and pitch angle of the spirals. This suggests that grand-design spirals in the inner kiloparsec can be explained by the propagation of gaseous density waves deep inside the ILR of the barred potential, when the right dynamical conditions are met. In particular, our study confirms that a low central mass concentration (evident through a shallow rotation curve), which may be common in many late-type galaxies, is particularly favorable to the propagation of bar-driven gaseous density waves deep into the central region of the galaxy. Conversely, a large central mass concentration favors other processes such as the formation and decoupling of nuclear bars.

Support for this work was generously provided by a grant from the K. T. and E. L. Norris Foundation, NSF grant AST 99-81546, and an AAUWEF Fellowship. I. S. acknowledges support under NAG 5-10823, HST GO08123.01-97A, and WKU 516140-02-07. We thank Dan Maoz, Aaron Barth, and Alex Filippenko for generously providing the $H S T$ continuum subtracted $\mathrm{H} \alpha+\left[\mathrm{N}_{\mathrm{II}}\right]$ images. We thank Bruce Elmegreen, Chi Yuan, and Frank Shu for interesting discussions. This publication is based on observations made with the NASA/ESA HST, obtained from the Data Archive at the Space Telescope Science Institute, which is operated by the Association of Universities for Research in Astronomy, Inc., under NASA contract NAS 5-26555. The Isaac Newton and William Herschel Telescopes are operated on the island of La Palma by the Isaac Newton Group (ING) in the Spanish Observatorio del Roque de los Muchachos of the Instituto de Astrofísica de Canarias. Data were partly retrieved from the ING archive.

\section{REFERENCES}

Aalto, S., Booth, R. S., Black, J. H., \& Johansson, L. E. B. 1995, A\&A, 300, 369

Allen, R. J., Atherton, P. D., \& Tilanus, R. P. J. 1986, Nature, 319, 296

Alonso-Herrero, A., Ryder, S. D., \& Knapen, J. H. 2001, MNRAS, 322, 757

Athanassoula, E. 1992, MNRAS, 259, 345

. 2002, ApJ, 569, L83

Bertin, G., Lin, C. C., Lowe, S. A., \& Thurstans, R. P. 1989a, ApJ, 338, 78 $1989 \mathrm{~b}, \mathrm{ApJ}, 338,104$

Binney, J., \& Tremaine, S. 1987, Galactic Dynamics (Princeton: Princeton Univ. Press)

Bloemen, J. B. G. M., et al. 1986, A\&A, 154, 25

Bureau, M. 2002, in ASP Conf. Ser., Disks of Galaxies: Kinematics, Dynamics, and Interactions, ed. E. Athanassoula \& A. Bosma (San Francisco: ASP), in press

Buta, R. 1984, Proc. Astron. Soc. Australia, 5, 472

Buta, R., \& Combes F. 1996, Fundam. Cosmic Phys., 17, 95

Buta, R., \& Crocker, D. 1993, AJ, 105, 1344

Buta, R., Treuthardt, P. M., Byrd, G. G., \& Crocker, D. A. 2000, AJ, 120, 1289

Combes, F., \& Gerin, M. 1985, A\&A, 150, 327

Condon, J. J. 1992, ARA\&A, 30, 575

Condon, J. J., Helou, G., Sanders, D. B., \& Soifer, T. B. 1990, ApJS, 73, 359

Contopoulos, G., \& Papayannopoulos, T. 1980, A\&A, 92, 33

de Vaucouleurs, G., de Vaucouleurs, A., Corwin, H. G., Jr., Buta, R. J., Paturel, G., \& Fouque, P. 1991, Third Reference Catalogue of Bright Galaxies (New York: Springer) (RC3)

Elmegreen, B. G. 1989, ApJ, 338, 178 1994, ApJ, 425, L73

Elmegreen, B. G., Seiden, P. E., \& Elmegreen, D. M. 1989, ApJ, 343, 602

Elmegreen, D. M., Chromey, F. R., Santos, M., \& Marshall, D. 1997, AJ, 114,1850

Englmaier, P., \& Shlosman, I. 2000, ApJ, 528, 677

Erwin, P., Beltran, J. C. V., \& Beckman, J. E. 2001, in ASP Conf. Ser. 249, The Central Kiloparsec of Starbursts and AGN: The La Palma Connection, ed. J. H. Knapen, J. E. Beckman, I. Shlosman, \& T. J. Mahoney (San Francisco: ASP), 171

Friedli, D., \& Martinet, L. 1993, A\&A, 277, 27

Helfer, T. T. \& Blitz, L. 1993, ApJ, 419, 86

Heller, C. H., \& Shlosman, I. 1996, ApJ, 471, 143

Heller, C. H., Shlosman, I., \& Englmaier, P. 2001, ApJ, 553, 661

Helou, G., Khan, I. R., Malek, L., Boehmer, L. 1988, ApJS, 68, 151

Ho, L. C., Filippenko, A. V., \& Sargent, W. L. W. 1997, ApJS, 112, 315

Hunter, D. A., O'Connell, R. W., Gallagher, J. S., \& Smecker-Hane, T. A. 2000, AJ, 120, 2383

Hüttemeister, S., \& Aalto, S. 2001, in ASP Conf. Ser. 249, The Central Kiloparsec of Starbursts and AGN: The La Palma Connection, ed. J. H. Knapen, J. E. Beckman, I. Shlosman, \& T. J. Mahoney (San Francisco: ASP), 619 
Jogee, S. 1999, Ph.D. thesis, Yale Univ. 2001, in Proc. Rinberg Workshop, Starbursts Near and Far, ed. L. Tacconi \& D. Lutz (Heidelberg: Springer), 182

Jogee, S., Baker, A. J., Sakamoto, K., Scoville, N. Z., \& Kenney, J. D. P. 2001, in ASP Conf. Ser. 249, The Central Kiloparsec of Starbursts and AGN: The La Palma Connection, ed. J. H. Knapen, J. E. Beckman, I. Shlosman, \& T. J. Mahoney (San Francisco: ASP), 612

Jogee, S., Kenney, J. D. P., \& Smith, B. J. 1999, ApJ, 526, 665

Jogee, S., Knapen, J. H., Laine, S., Shlosman, I., Scoville, N. Z., \& Englmaier, P. 2002, ApJ, 570, L55 (Paper I)

Kenney, J. D. P., Wilson, C. D., Scoville, N. Z., Devereux, N. A., \& Young, J. S. 1992, ApJ, 395, L79

Kenney, J. D. P., \& Young, J. S. 1989, ApJ, 344, 171

Kennicutt, R. C., Jr., Keel, W. C., \& Blaha, C. A. 1989, AJ, 97, 1022

Knapen, J. H., \& Beckman, J. E. 1996, MNRAS, 283, 251

Knapen, J. H., Beckman, J. E., Heller, C. H., Shlosman, I., \& de Jong, R. S. 1995a, ApJ, 454, 623

Knapen, J. H., Beckman, J. E., Shlosman, I., Peletier, R. F., Heller, C. H., \& de Jong, R. S. 1995b, ApJ, 443, L73

Knapen, J. H., Shlosman, I., Heller, C. H., Rand, R. J., Beckman, J. E., \& Rozas, M. 2000, ApJ, 528, 219

Koopmann, R. A. 1997, Ph.D. thesis, Yale Univ.

Laine, S., Kenney, J. D. P., Yun, M. S., \& Gottesman, S. T. 1999a, ApJ, 511,709

Laine, S., Knapen, J. H., Pérez-Ramírez, D., Doyon, R., \& Nadeau, D. 1999b, MNRAS, 302, L33

Laine, S., Knapen, J. H., Pérez-Ramírez, D., Englmaier, P., \& Matthias, M. 2001, MNRAS, 324, 891

Laine, S., Shlosman, I., Knapen, J. H., \& Peletier, R. F. 2002, ApJ, 567, 97

Leitherer, C., et al. 1999, ApJS, 123, 3

Leitherer, C., \& Heckman, T. M. 1995, ApJS, 96, 9

Lin, C. C., \& Shu, F. H. 1964, ApJ, 140, 646

Lin, C. C., Yuan, C., \& Shu, F. H. 1969, ApJ, 155, 721

Maciejewski, W., \& Sparke, L. S. 2000, MNRAS, 313, 745

Maíz-Apellániz, J., Muñoz-Tuñon, C., Tenorio-Tagle, G., \& Mas-Hesse, J. M. 1999, A\&A, 343, 64

Maoz, D., Barth, A. J., Ho, L. C., Sternberg, A., \& Filippenko, A. V. 2001, AJ, 121, 3048

Martin, P. 1995, AJ, 109, 2428

Martini, P., \& Pogge, R. W. 1999, AJ, 118, 2646

Noguchi, M. 1988, A\&A, 203, 259

Padin, S., Scott, S. L., Woody, D. P., Scoville, N. Z., Seling, T. V., Finch, R. P., Giovanine, C. J., \& Lawrence, R. P. 1991, PASP, 103, 461

Patsis, P., Grosbol, G., \& Hiotelis, N. 1997, A\&A, 326, 493

Pérez-Ramírez D., Knapen, J. H., Peletier, R. F., Laine, S., Doyon, R., \& Nadeau, D. 2000, MNRAS, 317, 234

Pfenniger, D. 1996, in Centennial Nobel Symp. on Barred Galaxies and Circumnuclear Activity, ed. A. Sandquist \& P. O. Lindblad (Berlin: Springer), 91
Regan, M. W., \& Mulchaey, J. S. 1999, AJ, 117, 2676

Regan, M. W., Thornley, M. D., Helfer, T. T., Sheth, K., Wong, T., Vogel, S. N., Blitz, L., \& Bock, D. C.-J. 2001, ApJ, 561, 218

Sakamoto, K., Okumura, S., Takeo, M., Yukiyasu, K., \& Wada, K. 1995, AJ, 110, 2075

Sakamoto, K., Okumura, S. K., Ishizuki, S., \& Scoville, N. Z. 1999, ApJS, 124,403

Schwarz, M. P. 1984, MNRAS, 221, 195

Scoville, N. Z., Carlstrom, J. E., Chandler, C. J., Phillips, J. A., Scott, S. L., Tilanus, R. P. J., \& Wang, Z. 1993, PASP, 105, 1982

Scoville, N. Z., \& Sanders, D. B. 1987, in Interstellar Processes, ed. D. J. Hollenbach \& H. A. Thronson (Dordrecht: Reidel), 21

Sheth, K. 2001, Ph.D. thesis, Univ. Maryland

Shlosman, I. 1996, in Centennial Nobel Symp. on Barred Galaxies and Circumnuclear Activity, ed. A. Sandqvist \& P. O. Lindblad (Berlin: Springer), 141

1999, in ASP Conf. Ser. 187, Evolution of Galaxies on Cosmological Timescales, ed. J. E. Beckman \& T. J. Mahoney (San Francisco: ASP), 100

. 2001, in ASP Conf. Ser. 249, The Central Kiloparsec of Starbursts and AGN: The La Palma Connection, ed. J. H. Knapen, J. E. Beckman, I. Shlosman, \& T. J. Mahoney (San Francisco: ASP), 55

2002, in ASP Conf. Ser., Disks of Galaxies: Kinematics, Dynamics, and Interactions, ed. E. Athanassoula \& A. Bosma (San Francisco: ASP), in press

Shlosman, I., Frank, J., \& Begelman, M. C. 1989, Nature, 338, 45

Shlosman, I., \& Heller, C. H. 2002, ApJ, 565, 921

Simkin, S. M., Su, H. J., \& Schwarz, M. P. 1980, ApJ, 237, 404

Sofue, Y. 1991, in IAU Symp. 146, Dynamics of Galaxies and Their Molecular Cloud Distributions, ed. F. Combes \& F. Casoli (Dordrecht: Kluwer), 156

Solomon, P. M., Rivolo, A. R., Barrett, J., \& Yahil, A. 1987, ApJ, 319, 730

Strickland, D. K., \& Stevens, I. R. 1999, MNRAS, 306, 43

Tilanus, R. P. J., \& Allen, R. J. 1989, ApJ, 339, L57 1991, A\&A, 244, 8

Tully, R. B. 1988, Nearby Galaxies Catalog (Cambridge: Cambridge Univ. Press)

Vanhala, H. A. T., \& Cameron, A. G. W. 1998, ApJ, 508, 291

Vila-Costas, M. B., \& Edmunds, M. G. 1992, MNRAS, 259, 121

Vogel, S. N., Kulkarni, S. R., \& Scoville, N. Z. 1988, Nature, 334, 402

Wall, W. F., \& Jaffe, D. T. 1990, ApJ, 361, L45

Whitmore, B. C., Zhang, Q., Leitherer, C., Fall, S. M., Schweizer, F., \& Miller, B. W. 1999, AJ, 118, 1551

Wild, W., Harris, A. I., Eckart, A., Genzel, R., Graf, U. U., Jackson, J. M.,

Russell, A. P. G., \& Stutzki, J. 1992, A\&A, 265, 447

Young, J. S., et al. 1995, ApJS, 98, 219 